\title{
The Kontsevich constants for the volume of the moduli of curves and topological recursion
}

Kevin M. Chapman, Motohico Mulase and Brad Safnuk

\begin{abstract}
We give an Eynard-Orantin-type topological recursion formula for the canonical Euclidean volume of the combinatorial moduli space of pointed smooth algebraic curves. The recursion comes from the edge removal operation on the space of ribbon graphs. As an application we obtain a new proof of the Kontsevich constants for the ratio of the Euclidean and the symplectic volumes of the moduli space of curves.
\end{abstract}

1. Introduction

2. The combinatorial model of the moduli space

3. Topological recursion for the number of integral ribbon graphs

4. The Laplace transform of the number of integral ribbon graphs

5. The Euclidean volume of the moduli space

6. The symplectic volume of the moduli space and the Kontsevich constants

7. The Eynard-Orantin theory on $\mathbb{P}^{1}$

Appendix A. calculation of the Laplace transforms 


\section{Introduction}

The purpose of this paper is to identify a combinatorial origin of the topological recursion formula of Eynard and Orantin [16] as the operation of edge removal from a ribbon graph. As an application of our formalism, we establish a new proof of the formula for the Kontsevich constants $\rho=2^{5 g-5+2 n}$ of [30, Appendix C].

In moduli theory it often happens that we have two different notions of the volume of the moduli space. The volume may be defined by the pushforward measure of the canonical construction of the moduli space. Or it may be defined as the symplectic volume with respect to the intrinsic symplectic structure of the moduli space. An example of such situations is the moduli space of flat $G$-bundles on a fixed Riemann surface for a compact Lie group $G[26,27,31,52]$. In this case, the two definitions of the volume agree.

The space we study in this paper is the combinatorial model of moduli space $\mathcal{M}_{g, n}$ of smooth algebraic curves of genus $g$ with $n$ distinct marked points. It also has two different families of volumes parametrized by $n$ positive real parameters. One comes from the push-forward measure, and the other comes from the intrinsic symplectic structure depending on these parameters. And again these two notions of volume agree.

The moduli space $\mathcal{M}_{g, n}$ admits orbifold cell-decompositions parametrized by the collection of positive real numbers assigned to the marked points. This orbifold is identified as the space of ribbon graphs of a prescribed perimeter length, using the theory of Strebel differentials. In his seminal paper of 1992, Kontsevich [30] calculated the symplectic volume of orbi-cells, and compared it with the standard Euclidean volume. He found that the ratio was a constant depending only on the genus of the curve and the number of marked points. This constant plays a crucial role in his main identity, and hence in his proof of the Witten conjecture. He wrote in Appendix C of [30] that his proof of the evaluation of this constant "presented here is not nice, but we do not know any other proof". In this article we give another proof of the formula for the Kontsevich constant, based on the topological recursion for ribbon graphs.

The idea of topological recursion (figure 1) has been used as an effective tool for calculating many quantities related to the moduli space $\mathcal{M}_{g, n}$ and its Deligne-Mumford compactification $\overline{\mathcal{M}}_{g, n}$. The quantities we can deal with include tautological intersection numbers and certain Gromov-Witten invariants. Suppose we have a collection of quantities $v_{g, n}$ for $g \geq 0$ and $n>0$ subject to the stability condition $2 g-2+n>0$, which guarantees the finiteness of the automorphism group of an element of $\mathcal{M}_{g, n}$. By an 


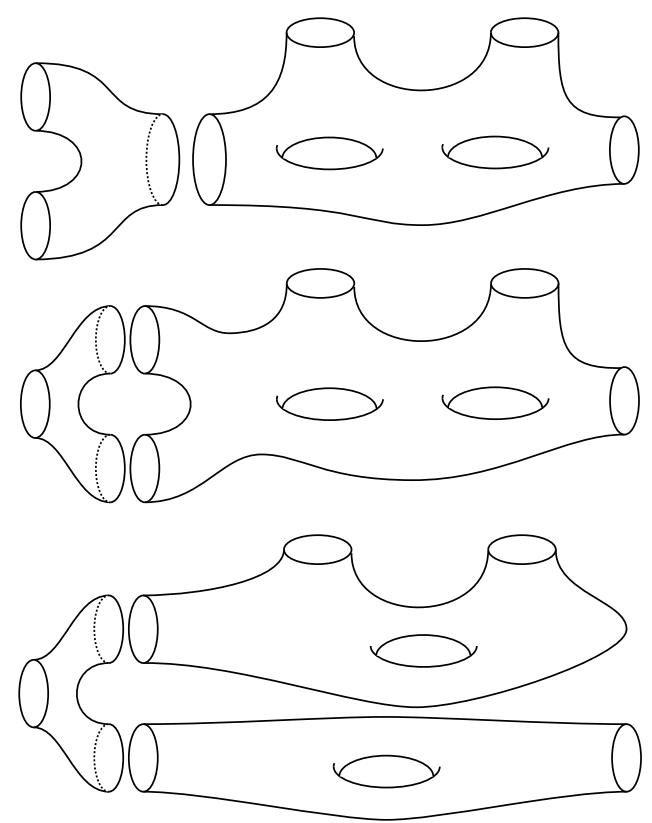

Figure 1: The topological recursion. The reduction of $2 g-2+n$ by 1 corresponds to cutting off of a pair of pants from an $n$-punctured surface.

Eynard-Orantin type topological recursion formula [16] we mean a particular inductive formula for $v_{g, n}$ with respect to the complexity $2 g-2+n$ of the form

$$
v_{g, n}=f_{1}\left(v_{g, n-1}\right)+f_{2}\left(v_{g-1, n+1}\right)+\sum_{\substack{g_{1}+g_{2}=g \\ n_{1}+n_{2}=n-1}}^{\text {stable }} f_{3}\left(v_{g_{1}, n_{1}+1}, v_{g_{1}, n_{2}+1}\right)
$$

with linear operators $f_{1}, f_{2}$ and a bilinear operator $f_{3}$, where the sum is taken for all possible partitions of $g$ and $n-1$ subject to the stability conditions $2 g_{1}-1+n_{1}>0$ and $2 g_{2}-1+n_{2}>0$. We refer to Section 7 for more details.

There are many examples of such formulas.

1. The Witten-Kontsevich theory for the tautological cotangent class (i.e., the $\psi$-class) intersection numbers

$$
\left\langle\tau_{d_{1}} \cdots \tau_{d_{n}}\right\rangle_{g, n}=\int_{\overline{\mathcal{M}}_{g, n}} c_{1}\left(\mathbb{L}_{1}\right)^{d_{1}} \cdots c_{1}\left(\mathbb{L}_{n}\right)^{d_{n}}
$$


on the moduli stack $\overline{\mathcal{M}}_{g, n}$ of stable algebraic curves of genus $g$ with $n$ distinct smooth marked points. The Dijkgraaf-Verlinde-Verlinde formula [9], which is equivalent to the Virasoro constraint condition, is a topological recursion of the form (1.1).

2. The Mirzakhani recursion formula for the Weil-Petersson volume of the moduli space of bordered hyperbolic surfaces with prescribed geodesic boundaries $[34,35]$ is a topological recursion.

3. Mixed intersection numbers

$$
\left\langle\tau_{d_{1}} \cdots \tau_{d_{n}} \kappa_{1}^{m_{1}} \kappa_{2}^{m_{2}} \kappa_{3}^{m_{3}} \cdots\right\rangle_{g, n}
$$

of $\psi$-classes and the Mumford-Morita-Miller $\kappa$-classes satisfy a topological recursion, first found in [37] for the case with $\kappa_{1}$ and later generalized in [32].

4. The expectation values of the product of resolvents of various matrix models satisfy a topological recursion (see, e.g., [13]). This is the origin of the work [16].

5. Indeed, the first three geometric theories turned out to be examples of the general theory [16] of topological recursions [14, 17], though geometric theories had been discovered earlier than the publication of [16].

6. Both open and closed Gromov-Witten invariants of an arbitrary toric Calabi-Yau three-fold are expected to satisfy a topological recursion. This is the remodeling conjecture of $[4,33]$.

7. Simple Hurwitz numbers satisfy a topological recursion. It was first conjectured in [5] based on a limit case of the remodeling conjecture, and was recently proved in $[3,15,38]$.

8. The simplest case of the remodeling conjecture for $\mathbb{C}^{3}$ was proved in $[7,53,54]$ based on the Laplace transform technique of [15].

9. As shown below, the number of metric ribbon graphs with integer edge lengths for a prescribed boundary condition satisfies a topological recursion.

Our current paper provides an elementary approach to the idea of topological recursion that uniformly explains the combinatorial nature of the geometric examples (1)-(3), (7)-(9). 
The work of Harer [23], Mumford [39], Strebel [48], and Thurston and others [47] show that there is a topological orbifold isomorphism

$$
\mathcal{M}_{g, n} \times \mathbb{R}_{+}^{n} \cong R G_{g, n}
$$

where

$$
R G_{g, n}=\coprod_{\substack{\Gamma \text { ribbon graph } \\ \text { of type }(g, n)}} \frac{\mathbb{R}_{+}^{e(\Gamma)}}{\operatorname{Aut}(\Gamma)}
$$

is the space of metric ribbon graphs of genus $g$ and $n$ boundary components, and $e(\Gamma)$ is the number of edges of a ribbon graph $\Gamma$. We denote by $\pi$ : $R G_{g, n} \longrightarrow \mathbb{R}_{+}^{n}$ the natural projection, and its fiber at $\mathbf{p} \in \mathbb{R}_{+}^{n}$ by $R G_{g, n}(\mathbf{p})=$ $\pi^{-1}(\mathbf{p})$. To give a combinatorial description of tautological intersection numbers (1.2) on $\overline{\mathcal{M}}_{g, n}$, Kontsevich [30,8] introduced a combinatorial symplectic form $\omega_{K}(\mathbf{p})$ on $R G_{g, n}(\mathbf{p}) \cong \mathcal{M}_{g, n}$ and its symplectic volume

$$
v_{g, n}^{\mathrm{S}}(\mathbf{p})=\int_{R G_{g, n}(\mathbf{p})} \exp \left(\omega_{K}(\mathbf{p})\right) .
$$

The definition of this symplectic form is given in Section 6. At each orbicell level, the derivative $d \pi$ of the projection map $\pi$ is determined by the edge-face incidence matrix

$$
A_{\Gamma}: \mathbb{R}_{+}^{e(\Gamma)} \longrightarrow \mathbb{R}_{+}^{n}
$$

of a ribbon graph $\Gamma$. Note that we have the standard volume forms $d \ell_{1} \wedge$ $\cdots \wedge d \ell_{e(\Gamma)}$ on $\mathbb{R}_{+}^{e(\Gamma)}$ and $d p_{1} \wedge \cdots \wedge d p_{n}$ on $\mathbb{R}_{+}^{n}$. We can define the Euclidean volume of the inverse image $P_{\Gamma}(\mathbf{p})=A_{\Gamma}^{-1}(\mathbf{p})$ of $\mathbf{p} \in \mathbb{R}_{+}^{n}$ using the pushforward measure by

$$
\operatorname{vol}\left(P_{\Gamma}(\mathbf{p})\right)=\left.\frac{\left(A_{\Gamma}\right)_{*}\left(d \ell_{1} \wedge \cdots \wedge d \ell_{e(\Gamma)}\right)}{d p_{1} \wedge \cdots \wedge d p_{n}}\right|_{\mathbf{p}},
$$

where $\left(A_{\Gamma}\right)_{*}\left(d \ell_{1} \wedge \cdots \wedge d \ell_{e(\Gamma)}\right)$ is the $n$-form on $\mathbb{R}_{+}^{n}$ obtained by integrating the volume form on $\mathbb{R}_{+}^{e(\Gamma)}$ along the fiber $A_{\Gamma}^{-1}(\mathbf{p})$. The Euclidean volume of the moduli space is defined by

$$
v_{g, n}^{\mathrm{E}}(\mathbf{p})=\sum_{\substack{\Gamma \text { ribbon graph } \\ \text { of type }(g, n)}} \frac{\operatorname{vol}\left(P_{\Gamma}(\mathbf{p})\right)}{|\operatorname{Aut}(\Gamma)|} .
$$

In Appendix C of [30], Kontsevich proved the following. 
Theorem 1.1 [30]. The ratio of the symplectic volume and the Euclidean volume of $R G_{g, n}(\mathbf{p})$ is a constant depending only on $g$ and $n$, and its value is

$$
\rho=\frac{v_{g, n}^{\mathrm{S}}(\mathbf{p})}{v_{g, n}^{\mathrm{E}}(\mathbf{p})}=2^{5 g-5+2 n} .
$$

Remark 1.1. The Euclidean volume of the polytope

$$
P_{\Gamma}(\mathbf{p})=\left\{\mathbf{x} \in \mathbb{R}_{+}^{e(\Gamma)} \mid A_{\Gamma} \mathbf{x}=\mathbf{p}\right\}
$$

is a quasi-polynomial and is difficult to calculate in general. It is quite surprising that the ratio $\rho$ of the two functions is indeed a constant. Although he says "not nice", Kontsevich's original proof is a beautiful application of homological algebra to the complexes defined by the incidence matrix $A_{\Gamma}$.

The new proof we present here uses an elementary argument on the topological recursion of ribbon graphs corresponding to the edge removal operation. We show that both $v_{g, n}^{\mathrm{S}}(\mathbf{p})$ and $2^{5 g-5+2 n} \cdot v_{g, n}^{\mathrm{E}}(\mathbf{p})$ satisfy exactly the same induction formula based on $2 g-2+n$, after taking the Laplace transform. We then calculate the initial condition for the recursion formula, i.e., the cases for $(g, n)=(0,3)$ and $(1,1)$, and see that the equality holds. Since the topological recursion uniquely determines the value for every $(g, n)$ subject to the stability condition $2 g-2+n>0$, we conclude that

$$
v_{g, n}^{\mathrm{S}}(\mathbf{p})=2^{5 g-5+2 n} \cdot v_{g, n}^{\mathrm{E}}(\mathbf{p}) .
$$

Here the appearance of the Laplace transform is significant. The Laplace transform plays a mysterious as well as a crucial role in each of the works $[14,15,17,30,38,45]$. In the light of the Eynard-Orantin recursion formalism [16] and the remodeling conjecture due to Mariño [33] and Bouchard-Klamm-Mariño-Pasquetti [4], we find that the Laplace transform appearing in these contexts is the mirror map. Usually mirror symmetry is considered as a duality, and hence a family of Fourier-Mukai type transforms naturally appears $[25,49]$. In our context, however, the nature of duality is not apparent. On one side of the story (the A-model side) we have a combinatorial structure. The mirror symmetry transforms this combinatorial structure into the world of complex analysis (the B-model side). In the complex analysis side we have such objects as the residue calculus of [16] and integrable nonlinear PDEs such as the $\mathrm{KdV}$ equations $[30,32,37,51]$, the KP hierarchy $[28,29,44]$, Frobenius manifold structures [11, 12], the 
Ablowitz-Ladik hierarchy [6] and more general integrable systems considered in [18-20]. The mathematical apparatus of the mirror map hidden in these structures is indeed the Laplace transform.

This paper is organized as follows. In Section 2 we review ribbon graphs and combinatorial description of the moduli space $\mathcal{M}_{g, n}$ that are necessary for our investigation. Although the definition of the Euclidean volume of $R G_{g, n}(\mathbf{p})$ is straightforward, it seems to be difficult to calculate it and there is no concrete formula. The approach we take in this paper is to appeal to the counting of lattice points of $R G_{g, n}(\mathbf{p})$. Thus Section 3 is devoted to proving an effective topological recursion formula for the number of lattice points in the space of metric ribbon graphs with prescribed perimeters. Our proof is based on counting ciliated ribbon graphs. Once we find the number of lattice points in $R G_{g, n}(\mathbf{p})$, we can obtain its volume by taking the limit as the mesh of the lattice tends to 0 . To compare the number of lattice points and the volume, the simplest path is to take the Laplace transform. Thus we are led to calculating the Laplace transform of the topological recursion for the number of lattice points in Section 4. After establishing the Laplace transform formula, one can read off the information of the Euclidean volume of $R G_{g, n}(\mathbf{p})$ as the leading terms of the Laplace transform, by introducing the right coordinate system. This is carried out in Section 5. The Kontsevich symplectic form is defined in Section 6, and the topological recursion for the symplectic volume due to [2] is reviewed. With these preparations, we give a new and simple proof of (1.4). In Section 7 we explain the Eynard-Orantin formalism. This formalism is independent on the context and provides the same formula. We then convert our recursion formulas into this formalism, and observe how they all fit together in a single formula. This is the beauty and strength of the Eynard-Orantin formalism.

We present a full detail of the calculations of the Laplace transform in this paper, hoping it may lead to a deeper understanding of the EynardOrantin theory and the mirror map. 7 is thus devoted to giving a proof of (4.6) and (6.11). These recursion formulas start with the initial values $(g, n)=(0,3)$ and $(g, n)=(1,1)$. The Eynard-Orantin theory also uses the unstable case $(g, n)=(0,2)$. All these values are calculated in 7 , together with a few more examples.

\section{The combinatorial model of the moduli space}

Let us begin with reviewing basic facts about ribbon graphs and the combinatorial model of the moduli space $\mathcal{M}_{g, n}$ due to Harer [23], Mumford [39] 
and Strebel [48]. We refer to [36] for precise definitions and more detailed exposition.

A ribbon graph of topological type $(g, n)$ is the 1-skeleton of a cell decomposition of a closed oriented topological surface $\Sigma$ of genus $g$ that decomposes the surface into a disjoint union of $v$ 0-cells, $e$ 1-cells, and $n$ 2 -cells. The Euler characteristic of the surface is given by $2-2 g=v-e+n$. The 1-skeleton of a cell decomposition is a graph $\Gamma$ drawn on $\Sigma$, which consists of $v$ vertices and $e$ edges. An edge can form a loop. We denote by $\Sigma_{\Gamma}$ the cell-decomposed surface with $\Gamma$ its 1-skeleton. Alternatively, a ribbon graph can be defined as a graph with a cyclic order given to the incident half-edges at each vertex. By abuse of terminology, we call the boundary of a two-cell of $\Sigma_{\Gamma}$ a boundary of $\Gamma$, and the two-cell itself as a face of $\Gamma$.

A metric ribbon graph is a ribbon graph with a positive real number (the length) assigned to each edge. For a given ribbon graph $\Gamma$ with $e=e(\Gamma)$ edges, the space of metric ribbon graphs is $\mathbb{R}_{+}^{e(\Gamma)} / \operatorname{Aut}(\Gamma)$, where the automorphism group acts through permutations of edges (see [36, Section 1]). We restrict ourselves to the case that $\operatorname{Aut}(\Gamma)$ fixes each two cell of the cell decomposition. If we also restrict that every vertex of a ribbon graph has degree (i.e., valence) 3 or more, then using the canonical holomorphic coordinate system of a topological surface [36, Section 4] and the Strebel differentials [48], we obtain an isomorphism of topological orbifolds [23,39,47]

$$
\mathcal{M}_{g, n} \times \mathbb{R}_{+}^{n} \cong R G_{g, n}
$$

Here

$$
R G_{g, n}=\coprod_{\substack{\Gamma \text { ribbon graph } \\ \text { of type }(g, n)}} \frac{\mathbb{R}_{+}^{e(\Gamma)}}{\operatorname{Aut}(\Gamma)}
$$

is the orbifold consisting of metric ribbon graphs of a given topological type $(g, n)$ with degree 3 or more. The degree condition is necessary to bound the number of edges $e(\Gamma)$ for a given topological type $(g, n)$. If we allow degree 2 vertices, then there are infinitely many different ribbon graphs for every $(g, n)$. By restricting to ribbon graphs of degree 3 or more, we have the bound $e(\Gamma) \leq 3(2 g-2+n)$, which gives the dimension of each orbi-cell $\mathbb{R}_{+}^{e(\Gamma)} / \operatorname{Aut}(\Gamma)$. The gluing of orbi-cells is done by making the length of a non-loop edge tend to 0 . The space $R G_{g, n}$ is a smooth orbifold (see [36, Section 3]; [47]). We denote by $\pi: R G_{g, n} \longrightarrow \mathbb{R}_{+}^{n}$ the natural projection via (2.1), which is the assignment of the collection of perimeter length of each boundary to a given metric ribbon graph. 
Take a ribbon graph $\Gamma$. Since Aut $(\Gamma)$ fixes every boundary component of $\Gamma$, they can be labeled by $N=\{1,2, \ldots, n\}$. For a moment let us give a label to each edge of $\Gamma$ from an index set $E=\{1,2, \ldots, e\}$. The edge-face incidence matrix is defined by

$$
\begin{aligned}
& A_{\Gamma}=\left[a_{i \eta}\right]_{i \in N, \eta \in E}, \\
& a_{i \eta}=\text { the number of times edge } \eta \text { appears in face } i .
\end{aligned}
$$

Thus $a_{i \eta}=0,1$, or 2 , and the sum of entries in each column is always 2 . The $\Gamma$ contribution of the space $\pi^{-1}\left(p_{1}, \ldots, p_{n}\right)=R G_{g, n}(\mathbf{p})$ of metric ribbon graphs with a prescribed perimeter $\mathbf{p}=\left(p_{1}, \ldots, p_{n}\right)$ is the orbifold polytope

$$
P_{\Gamma}(\mathbf{p}) / \operatorname{Aut}(\Gamma), \quad P_{\Gamma}(\mathbf{p})=\left\{\mathbf{x} \in \mathbb{R}_{+}^{e} \mid A_{\Gamma} \mathbf{x}=\mathbf{p}\right\}
$$

where $\mathbf{x}=\left(\ell_{1}, \ldots, \ell_{e}\right)$ is the collection of edge lengths of a metric ribbon graph $\Gamma$. We have

$$
\sum_{i \in N} p_{i}=\sum_{i \in N} \sum_{\eta \in E} a_{i \eta} \ell_{\eta}=2 \sum_{\eta \in E} \ell_{\eta}
$$

The canonical Euclidean volume $\operatorname{vol}\left(P_{\Gamma}(\mathbf{p})\right)$ of the polytope $P_{\Gamma}(\mathbf{p})$ is the ratio of the push-forward measure of the Lebesgue measure on $\mathbb{R}_{+}^{e}$ by $A_{\Gamma}$ and the Lebesgue measure on $\mathbb{R}_{+}^{n}$ at the point $\mathbf{p} \in \mathbb{R}_{+}^{n}$ :

$$
\operatorname{vol}\left(P_{\Gamma}(\mathbf{p})\right)=\left.\frac{\left(A_{\Gamma}\right)_{*}\left(d \ell_{1} \wedge \cdots \wedge d \ell_{e}\right)}{d p_{1} \wedge \cdots \wedge d p_{n}}\right|_{\mathbf{p}},
$$

where $\left(A_{\Gamma}\right)_{*}\left(d \ell_{1} \wedge \cdots \wedge d \ell_{e}\right)$ is the $n$-form on $\mathbb{R}_{+}^{n}$ obtained by integrating the volume form on $\mathbb{R}_{+}^{e}$ along the fiber $\pi^{-1}(\mathbf{p})$. This definition is equivalent to imposing

$$
\int_{D} \operatorname{vol}\left(P_{\Gamma}(\mathbf{p})\right) d p_{1} \wedge \cdots \wedge d p_{n}=\int_{A_{\Gamma}^{-1}(D)} d \ell_{1} \wedge \cdots \wedge d \ell_{e}
$$

for every open subset $D \subset \mathbb{R}_{+}^{n}$ with compact closure. We define the Euclidean volume function by

$$
v_{g, n}^{\mathrm{E}}(\mathbf{p})=v_{g, n}^{\mathrm{E}}\left(p_{1}, \ldots, p_{n}\right)=\sum_{\substack{\Gamma \text { trivalent ribbon } \\ \text { graph of type }(g, n)}} \frac{\operatorname{vol}\left(P_{\Gamma}(\mathbf{p})\right)}{|\operatorname{Aut}(\Gamma)|} .
$$


This is the Euclidean volume of the moduli space $\mathcal{M}_{g, n}$ considered as the orbi-cell complex

$$
R G_{g, n}(\mathbf{p}) \stackrel{\text { def }}{=} \pi^{-1}(\mathbf{p})=\coprod_{\substack{\Gamma \text { ribbon graph } \\ \text { of type }(g, n)}} \frac{P_{\Gamma}(\mathbf{p})}{\operatorname{Aut}(\Gamma)} \cong \mathcal{M}_{g, n}
$$

with the prescribed perimeter length $\mathbf{p} \in \mathbb{R}_{+}^{n}$. Only degree 3 (or trivalent) graphs contribute to the volume function because they parametrize the top dimensional cells. Since $\operatorname{dim}_{\mathbb{R}} R G_{g, n}(\mathbf{p})=2(3 g-3+n)$, we expect that the definition of the push-forward measure and the relation (2.5) imply that the volume function $v_{g, n}^{\mathrm{E}}(\mathbf{p})$ has the polynomial growth of order $2(3 g-3+n)$ as $\mathbf{p} \rightarrow \infty$. We will verify this growth order in Section 5 (5.3).

\section{Topological recursion for the number of integral ribbon graphs}

It is a difficult task to find a topological recursion formula for the Euclidean volume functions $v_{g, n}^{\mathrm{E}}(\mathbf{p})$ directly from its definition. One might think that the Weil-Petersson volume of the moduli of bordered hyperbolic surfaces $[34,35]$ would give the Euclidean volume at the long boundary limit, but actually the limit naturally converges to the symplectic volume we consider in Section 6. The straightforward method for the Euclidean volume is indeed to go through the detour of considering the lattice point counting. We therefore first derive a recursion formula for the number of metric ribbon graphs with integer edge lengths, take its Laplace transform, and then extract the topological recursion for the Euclidean volume functions.

Thus our main subject of this section is the set of all metric ribbon graphs $R G_{g, n}^{\mathbb{Z}_{+}}$whose edges have integer lengths. We call such a ribbon graph an integral ribbon graph. Following [40], let us define the weighted number $\left|R G_{g, n}^{\mathbb{Z}_{+}}(\mathbf{p})\right|$ of integral ribbon graphs with prescribed perimeter lengths $\mathbf{p} \in$ $\mathbb{Z}_{+}^{n}$ (figure 2):

$$
N_{g, n}(\mathbf{p})=\left|R G_{g, n}^{\mathbb{Z}_{+}}(\mathbf{p})\right|=\sum_{\substack{\Gamma \text { ribbon graph } \\ \text { of type }(g, n)}} \frac{\left|\left\{\mathbf{x} \in \mathbb{Z}_{+}^{e(\Gamma)} \mid A_{\Gamma} \mathbf{x}=\mathbf{p}\right\}\right|}{|\operatorname{Aut}(\Gamma)|} .
$$

Since the finite set $\left\{\mathbf{x} \in \mathbb{Z}_{+}^{e(\Gamma)} \mid A_{\Gamma} \mathbf{x}=\mathbf{p}\right\}$ is a collection of lattice points in the polytope $P_{\Gamma}(\mathbf{p})$ with respect to the canonical integral structure $\mathbb{Z} \subset \mathbb{R}$ of the real numbers, $N_{g, n}(\mathbf{p})$ can be thought of as counting the number of lattice 


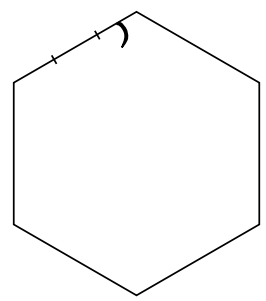

Figure 2: A ciliation in a face. The cilium is placed on a bordering edge, 0.5 unit length away from the nearest vertex.

points in $R G_{g, n}(\mathbf{p})$ with a weight factor $1 /|\operatorname{Aut}(\Gamma)|$ for each ribbon graph. The function $N_{g, n}(\mathbf{p})$ is a symmetric function in $\mathbf{p}=\left(p_{1}, \ldots, p_{n}\right)$ because the summation runs over all ribbon graphs of topological type $(g, n)$ whose boundaries are labeled by the index set $N$.

Remark 3.1. The function (3.1) was first considered in [40]. Note that we do not allow the integer vector $\mathbf{p} \in \mathbb{Z}_{+}^{n}$ to have any 0 entry, since each face of a ribbon graph must have a positive perimeter length. Note that $A_{\Gamma} \mathbf{x}=0$ has no positive solutions. Therefore, the natural extension of the definition (3.1) to the case of $\mathbf{p}=0$ would give $N_{g, n}(0)=0$.

Using the lattice point interpretation, it is easy to see that the relation between this function and the Euclidean volume function is the same as that of the Riemann sum and the Riemann integral. Let $k$ be a positive integer and $D \subset \mathbb{R}_{+}^{n}$ an open domain with compact closure. Then for every continuous function $f(\mathbf{p})$ on $D$, the definition of the Riemann integration in terms of Riemann sums gives

$$
\lim _{k \rightarrow \infty} \sum_{\mathbf{p} \in D \cap \frac{1}{k} \mathbb{Z}_{+}^{n}} N_{g, n}(k \mathbf{p}) f(\mathbf{p}) \frac{1}{k^{3(2 g-2+n)}}=\int_{D} v_{g, n}^{\mathrm{E}}(\mathbf{p}) f(\mathbf{p}) d p_{1} \cdots d p_{n} .
$$

This equality holds because our definition of the volume uses the pushforward measure. We note that as a function in $\mathbf{p}$ there is no simple direct relation between the values $N_{g, n}(\mathbf{p})$ and $v_{g . n}^{\mathrm{E}}(\mathbf{p})$. For example, $N_{g, n}(\mathbf{p})=0$ if $\sum_{i=1}^{n} p_{i}$ is odd because of $(2.3)$, but the volume function is not subject to such a relation.

To derive a topological recursion for $N_{g, n}(\mathbf{p})$, we introduce the notion of ciliation.

Definition 3.1. A ciliation is an assignment of a cilium in a face attached to a bordering edge (see figure 2 ). Let $\ell \in \mathbb{Z}_{+}$be the length of the edge on 
which the ciliation is attached. We place the root of the cilium at a halfinteger length away from the vertices bounding the edge. Thus no cilium is attached to a vertex of a ribbon graph.

The number of ciliations of a metric ribbon graph $\Gamma$ with integer edge lengths is given by (2.3). Indeed, if we count with respect to the edges, then there are $2 \ell$ ways for a ciliation to each edge because the cilium can be placed on each side of the edge. And each face $i$ has $p_{i}$ ways of ciliation. Thus the total number of ciliations is $p_{1}+\cdots+p_{n}$.

For brevity of notation, we denote by $p_{I}=\left(p_{i}\right)_{i \in I}$ for a subset $I \in N=$ $\{1,2, \ldots, n\}$. The cardinality of $I$ is denoted by $|I|$.

Theorem 3.1. The number of integral metric ribbon graphs with prescribed boundary lengths satisfies the following topological recursion formula:

$$
\begin{aligned}
p_{1} N_{g, n}\left(p_{N}\right)= & \frac{1}{2} \sum_{j=2}^{n}\left[\sum_{q=0}^{p_{1}+p_{j}} q\left(p_{1}+p_{j}-q\right) N_{g, n-1}\left(q, p_{N \backslash\{1, j\}}\right)\right. \\
& +H\left(p_{1}-p_{j}\right) \sum_{q=0}^{p_{1}-p_{j}} q\left(p_{1}-p_{j}-q\right) N_{g, n-1}\left(q, p_{N \backslash\{1, j\}}\right) \\
& \left.-H\left(p_{j}-p_{1}\right) \sum_{q=0}^{p_{j}-p_{1}} q\left(p_{j}-p_{1}-q\right) N_{g, n-1}\left(q, p_{N \backslash\{1, j\}}\right)\right] \\
& +\frac{1}{2} \sum_{\substack{0 \leq q_{1}+q_{2} \leq p_{1} \\
\text { stable }}} q_{1} q_{2}\left(p_{1}-q_{1}-q_{2}\right)\left[N_{g-1, n+1}\left(q_{1}, q_{2}, p_{N \backslash\{1\}}\right)\right. \\
& \left.+\sum_{\substack{g_{1}+g_{2}=g \\
I \sqcup J=N \backslash\{1\}}} N_{g_{1},|I|+1}\left(q_{1}, p_{I}\right) N_{g_{2},|J|+1}\left(q_{2}, p_{J}\right)\right]
\end{aligned}
$$

Here

$$
H(x)= \begin{cases}1 & x>0 \\ 0 & x \leq 0\end{cases}
$$

is the Heaviside function, and the last sum is taken for all partitions $g=$ $g_{1}+g_{2}$ and $I \sqcup J=N \backslash\{1\}$ subject to the stability condition $2 g_{1}-1+I>0$ and $2 g_{2}-1+|J|>0$. 
Proof. The key idea is to count all integral ribbon graphs with a cilium placed on the face named 1 . The number is clearly equal to $p_{1} N_{g, n}\left(p_{N}\right)$. We then analyze what happens when we remove the ciliated edge from the ribbon graph. There are several situations after the removal of this edge. The right-hand side of the recursion formula is obtained by the case-by-case analysis of the edge removal operation. For any ciliated ribbon graph of type $(g, n)$ subject to the condition $2 g-2+n>1$, removing the ciliated edge creates a new graph of type $(g, n-1)$ or $(g-1, n+1)$, or two disjoint graphs of types $\left(g_{1}, n_{1}+1\right)$ and $\left(g_{2}, n_{2}+1\right)$ subject to the stability condition and the partition condition

$$
\left\{\begin{array}{l}
g_{1}+g_{2}=g \\
n_{1}+n_{2}=n-1
\end{array}\right.
$$

Note that in each case the quantity $2 g-2+n$ is reduced exactly by 1 .

Let $\eta$ be the edge bordering face 1 of a ribbon graph $\Gamma$ on which the cilium is placed, and $a_{1 \eta}$ the incidence number of (2.2). Let $\ell \in \mathbb{Z}_{+}$be the length of edge $\eta$. There are two main situations: $a_{1 \eta}=1$ and $a_{1 \eta}=2$. Each main situation breaks down further into three cases. Before examining each care in detail, we first need to analyze the effect of $\operatorname{Aut}(\Gamma)$ in the edge removal operation. Note that the automorphism group fixes each face. Thus $\eta$ moves to another edge $\eta^{\prime}$ of face 1 . If $\eta=\eta^{\prime}$, then the automorphism is unaffected by the edge removal and we have $\operatorname{Aut}(\Gamma)=\operatorname{Aut}(\Gamma \backslash \eta)$, where the right-hand side is a product group if $\Gamma \backslash \eta$ is disconnected. If $\eta \neq \eta^{\prime}$, then placing a cilium on $\eta$ or $\eta^{\prime}$ inside face 1 is indistinguishable, and this identification is accounted for in the counting $p_{1} N_{g, n}\left(p_{N}\right)$.

Case 1. $a_{1 \eta}=a_{j \eta}=1$ for $j \geq 2, p_{1}>\ell$ and $p_{j}>\ell$. Define $q=\left(p_{1}-\ell\right)+$ $\left(p_{j}-\ell\right)>0$. Then we have

$$
\left\{\begin{array}{l}
q-p_{1}+p_{j}=2\left(p_{j}-\ell\right)>0 \\
q+p_{1}-p_{j}=2\left(p_{1}-\ell\right)>0
\end{array}\right.
$$

Therefore, $q>\left|p_{1}-p_{j}\right|$. Geometrically, $q$ is the perimeter length of the face created by removing edge $\eta$ that separates faces 1 and $j$ (see figure 3 ).

To recover the original ribbon graph with a cilium on edge $\eta$ of length $\ell$ from the one without edge $\eta$, we need to place the edge on the face of perimeter $q$, and place a cilium on this edge. Here we note that the data $p_{i}, p_{j}, q$ and $\ell$ are all prescribed. The number of ways to place an endpoint of the edge on the face of perimeter length $q$ is $q$. This point uniquely 


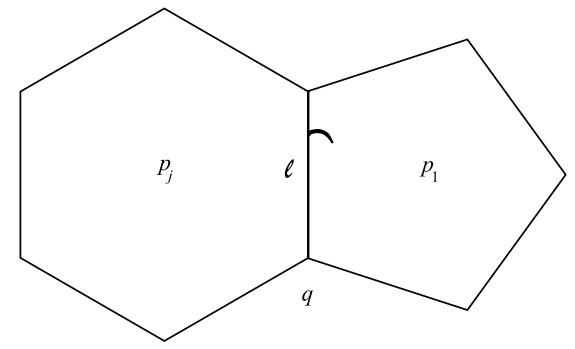

Figure 3: Case 1: $a_{1 \eta}=a_{j \eta}=1, p_{1}>\ell$ and $p_{j}>\ell$.

determines the edge we need, since the other endpoint is $p_{1}-\ell$ away from the first endpoint along the perimeter measured by the clockwise distance. The enclosed face of perimeter length $p_{1}$ becomes face 1 , and the other side of the newly placed edge is face $j$. Since the ciliation is done on face 1 , there are $\ell$ choices for the assignment of the root of the cilium. Altogether, the contribution of this case is

$$
\sum_{q=\left|p_{1}-p_{j}\right|+1}^{p_{1}+p_{j}} q \frac{p_{1}+p_{j}-q}{2} N_{g, n-1}\left(q, p_{N \backslash\{1, j\}}\right) .
$$

Case 2. $a_{1 \eta}=a_{j \eta}=1$ for $j \geq 2$, and $p_{1} \geq p_{j}=\ell$. Since $p_{j}=\ell$, face $j$ and edge $\eta$ are the same and forms a loop. This loop is connected to face 1 by an edge $\eta^{\prime}$ of incidence number 2 . Let $\ell^{\prime}$ be the length of this connecting edge, which is bounded by $\left(p_{1}-p_{j}\right) / 2 \geq \ell^{\prime} \geq 0$ (see figure 4 , left). This time define $q=p_{1}-p_{j}-2 \ell^{\prime}$. This is the perimeter length of the face created by removing face $j$ and edge $\eta^{\prime}$. In this situation, removing edge $\eta$ (= face $j$ ) alone does not create an admissible ribbon graph, since edge $\eta^{\prime}$ remains with a vertex of degree 1 at one end. Therefore, we need to remove the entire tadpole consisting of a head of face $j$ and a tail of edge $\eta^{\prime}$. The cilium is on face 1 , which is attached to the outer boundary of face $j$.

To recover the original graph from the result of this tadpole removal, we have $q$ choices for the tadpole placement and $p_{j}=\ell$ choices for ciliation. Therefore, the contribution from this case is

$$
\sum_{q=0}^{p_{1}-p_{j}} q p_{j} N_{g, n-1}\left(q, p_{N \backslash\{1, j\}}\right) .
$$

Case 3. $a_{1 \eta}=a_{j \eta}=1$ for $j \geq 2$, and $p_{j} \geq p_{1}=\ell$. The situation is similar to Case 2 (see figure 4 , center). Let $\eta^{\prime}$ be the edge of length $\ell^{\prime}$ that connects 

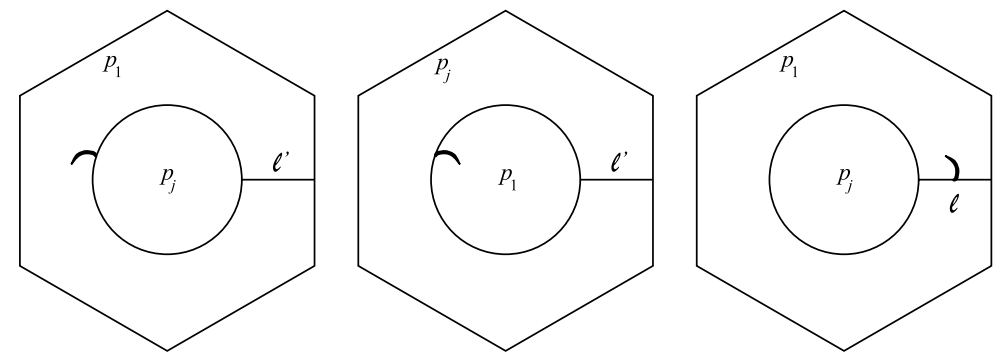

Figure 4: Case 2 (left): $a_{1 \eta}=a_{j \eta}=1$ and $p_{1} \geq p_{j}=\ell$; Case 3 (center): $a_{1 \eta}=$ $a_{j \eta}=1$ and $p_{j} \geq p_{1}=\ell$; and Case 4 (right): $a_{1 \eta}=2$ and the edge $\eta$ connects a loop $j$ to the rest of the graph.

face 1 and face $j$. Define $q=p_{j}-p_{1}-2 \ell^{\prime}$. This is the perimeter length of the face created by removing the entire tadpole consisting of face 1 with a cilium as its head and edge $\eta^{\prime}$ as its tail. We have $q$ choices for tadpole placement and $p_{1}$ choices for ciliation. Thus the contribution is

$$
\sum_{q=0}^{p_{j}-p_{1}} q p_{1} N_{g, n-1}\left(q, p_{N \backslash\{1, j\}}\right) .
$$

Case 4. $a_{1 \eta}=2$ and removal of edge $\eta$ separates a single loop $j$ for some $j \geq 2$ from the rest of the graph (see figure 4 , right). It is necessary that $p_{1}>$ $p_{j}$ in this case. Since a single loop alone is not an admissible graph, we need to remove face $j$ together when we remove edge $\eta$. Define $q=p_{1}-p_{j}-2 \ell$, which is the perimeter length of the face created after the removal of the tadpole. This time the recovery process has $q$ choices of tadpole placement and $2 \ell$ choices for ciliation, because the cilium can be placed on either side of the tail. Thus the contribution is

$$
\sum_{q=0}^{p_{1}-p_{j}} q\left(p_{1}-p_{j}-q\right) N_{g, n-1}\left(q, p_{N \backslash\{1, j\}}\right) .
$$

Case 5. $a_{1 \eta}=2$ and removal of edge $\eta$ creates a connected ribbon graph. The removal of edge $\eta$ breaks face 1 into two separate faces of perimeter lengths $q_{1}$ and $q_{2}$ subject to the condition $0<q_{1}+q_{2}<p_{1}$. The removal of the edge reduces the genus by 1 , and increases the number of faces by 1 . We have the equality $p_{1}=q_{1}+q_{2}+2 \ell$ (see figure 5 ). To recover the original graph from the result of the edge removal, we have $q_{1}$ choices for one endpoint of edge $\eta, q_{2}$ choices for the other endpoint, and $2 \ell$ choices for ciliation, again because the cilium can be placed on either side of edge $\eta$. 


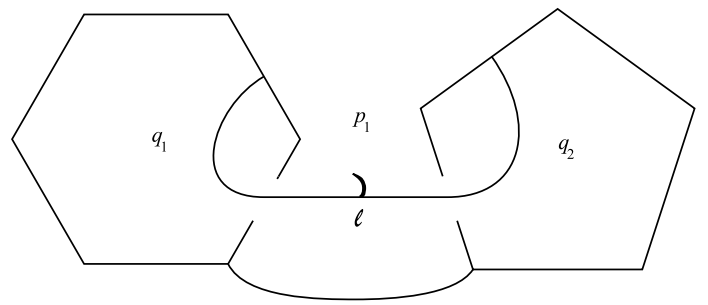

Figure 5: Case 5: $a_{1 \eta}=2$ and removal of edge $\eta$ creates a connected ribbon graph.

Altogether the contribution is

$$
\frac{1}{2} \sum_{0 \leq q_{1}+q_{2} \leq p_{1}} q_{1} q_{2}\left(p_{1}-q_{1}-q_{2}\right) N_{g-1, n+1}\left(q_{1}, q_{2}, p_{N \backslash\{1\}}\right) .
$$

Here we need the factor $\frac{1}{2}$, which is the symmetry factor of interchanging $q_{1}$ and $q_{2}$.

Case 6. $a_{1 \eta}=2$ and removal of edge $\eta$ creates a disjoint union of two ribbon graphs. There are $n$ faces in the original ribbon graph $\Gamma$. The removal of edge $\eta$ breaks face 1 into two separate faces of perimeter lengths $q_{1}$ and $q_{2}$. The other faces $2,3, \ldots, n$ remain intact. Let $I \subset N \backslash\{1\}$ be the label of faces that are connected to the new face of perimeter length $q_{1}$, and $J \subset N \backslash\{1\}$ for $q_{2}$. Then the two disjoint ribbon graphs have types $\left(g_{1},|I|+1\right)$ and $\left(g_{2},|J|+1\right)$ satisfying the partition condition (figure 6$)$ :

$$
\left\{\begin{array}{l}
g_{1}+g_{2}=g \\
I \sqcup J=N \backslash\{1\} .
\end{array}\right.
$$

The contribution from this case is

$$
\frac{1}{2} \sum_{0 \leq q_{1}+q_{2} \leq p_{1}} q_{1} q_{2}\left(p_{1}-q_{1}-q_{2}\right) \sum_{\substack{g_{1}+g_{2}=g \\ I \sqcup J=N \backslash\{1\}}}^{\text {stable }} N_{g_{1},|I|+1}\left(q_{1}, p_{I}\right) N_{g_{2},|J|+1}\left(q_{2}, p_{J}\right)
$$

with the symmetry factor $\frac{1}{2}$ corresponding to interchanging $q_{1}$ and $q_{2}$.

Summing all contributions (3.4)-(3.9), we obtain

$$
p_{1} N_{g, n}\left(p_{N}\right)=\sum_{j=2}^{n} \sum_{q=\left|p_{1}-p_{j}\right|+1}^{p_{1}+p_{j}} q \frac{p_{1}+p_{j}-q}{2} N_{g, n-1}\left(q, p_{N \backslash\{1, j\}}\right)
$$




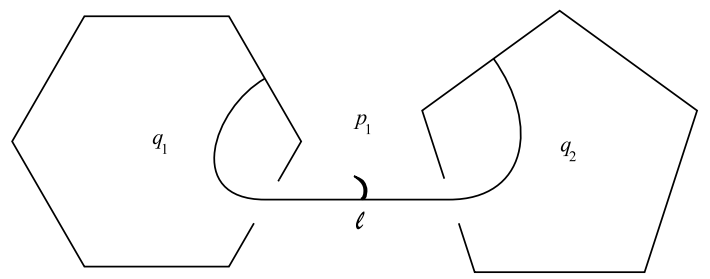

Figure 6: Case 6: $a_{1 \eta}=2$ and removal of edge $\eta$ creates a disjoint union of two ribbon graphs.

$$
\begin{aligned}
& +\sum_{j=2}^{n} H\left(p_{1}-p_{j}\right) \sum_{q=0}^{p_{1}-p_{j}} q\left(p_{1}-q\right) N_{g, n-1}\left(q, p_{N \backslash\{1, j\}}\right) \\
& +\sum_{j=2}^{n} H\left(p_{j}-p_{1}\right) \sum_{q=0}^{p_{j}-p_{1}} q p_{1} N_{g, n-1}\left(q, p_{N \backslash\{1, j\}}\right) \\
& +\frac{1}{2} \sum_{0 \leq q_{1}+q_{2} \leq p_{1}} q_{1} q_{2}\left(p_{1}-q_{1}-q_{2}\right)\left[N_{g-1, n+1}\left(q_{1}, q_{2}, p_{N \backslash\{1\}}\right)\right. \\
& \left.+\sum_{\substack{\text { stable } \\
g_{1}+g_{2}=g \\
I \sqcup J=N \backslash\{1\}}} N_{g_{1},|I|+1}\left(q_{1}, p_{I}\right) N_{g_{2},|J|+1}\left(q_{2}, p_{J}\right)\right]
\end{aligned}
$$

If we allow the variable $q$ to range from 0 to $p_{1}+p_{j}$ in the first summation of the right-hand side of (3.10), then we need to compensate the non-existing cases. Note that we have

$$
\begin{aligned}
& -\sum_{j=2}^{n} \sum_{q=0}^{\left|p_{1}-p_{j}\right|} q \frac{p_{1}+p_{j}-q}{2} N_{g, n-1}\left(q, p_{N \backslash\{1, j\}}\right) \\
& +\sum_{j=2}^{n} H\left(p_{1}-p_{j}\right) \sum_{q=0}^{p_{1}-p_{j}} q \frac{2 p_{1}-2 q}{2} N_{g, n-1}\left(q, p_{N \backslash\{1, j\}}\right) \\
& +\sum_{j=2}^{n} H\left(p_{j}-p_{1}\right) \sum_{q=0}^{p_{j}-p_{1}} q \frac{2 p_{1}}{2} N_{g, n-1}\left(q, p_{N \backslash\{1, j\}}\right) \\
& \quad=\sum_{j=2}^{n} H\left(p_{1}-p_{j}\right) \sum_{q=0}^{p_{1}-p_{j}} q \frac{p_{1}-p_{j}-q}{2} N_{g, n-1}\left(q, p_{N \backslash\{1, j\}}\right)
\end{aligned}
$$




$$
-\sum_{j=2}^{n} H\left(p_{j}-p_{1}\right) \sum_{q=0}^{p_{j}-p_{1}} q \frac{p_{j}-p_{1}-q}{2} N_{g, n-1}\left(q, p_{N \backslash\{1, j\}}\right) .
$$

Substituting (3.11) in (3.10), we obtain (3.3). This completes the proof.

Remark 3.2. The topological recursion for $N_{g, n}(\mathbf{p})$ was first considered by Norbury in [40]. His proof is similar in that it involved an edge removal operation, but the main formula and its proof therein contained are incorrectly recorded - the terms involving products of functions $N_{g, n}$ were double counted and need a compensating factor of $\frac{1}{2}$. A corrected version appears in $[10,42]$. Our proof presented here is new, and is based on a different idea using ciliation.

\section{The Laplace transform of the number of integral ribbon graphs}

The limit formula (3.2) tells us that $N_{g, n}(\mathbf{p})$ asymptotically behaves like a polynomial for large $\mathbf{p} \in \mathbb{Z}_{+}^{n}$, and the coefficients of the leading terms correspond to that of the Euclidean volume function $v_{g, n}^{\mathrm{E}}(\mathbf{p})$. The lack of the direct relation between $N_{g, n}(\mathbf{p})$ and $v_{g, n}^{\mathrm{E}}(\mathbf{p})$, together with Equation (3.2), suggest that we need to consider an integral transform, such as the Laplace transform of $N_{g, n}(\mathbf{p})$, to extract the information of the Euclidean volume of $R G_{g, n}(\mathbf{p})$ from it. Since

$$
\int_{0}^{\infty} x^{m} \mathrm{e}^{-x w} d x=\frac{m !}{w^{m+1}}
$$

for a complex variable $w \in \mathbb{C}$ with $\operatorname{Re}(w)>0$, the coefficients of the highest order poles of the Laplace transform

$$
L_{g, n}\left(w_{1}, \ldots, w_{n}\right) \stackrel{\text { def }}{=} \sum_{\mathbf{p} \in \mathbb{Z}_{+}^{n}} N_{g, n}(\mathbf{p}) \mathrm{e}^{-\langle\mathbf{p}, w\rangle}
$$

should represent the Euclidean volume of $R G_{g, n}(\mathbf{p})$. Here $\langle p, w\rangle=$ $p_{1} w_{1}+\cdots+p_{n} w_{n}$. This section is devoted to the analysis of the Laplace transform of the topological recursion (3.3).

To relate our investigation with the Hurwitz theory and the WittenKontsevich theory, and in particular from the point of view of the polynomial 
expressions of $[15,38]$, we introduce new complex coordinates

$$
\mathrm{e}^{-w}=\frac{t+1}{t-1} \quad \text { and } \quad \mathrm{e}^{-w_{j}}=\frac{t_{j}+1}{t_{j}-1}
$$

and express the result of the Laplace transform in terms of these $t$-variables. This substitution makes sense because the Laplace transform is a rational function in $\mathrm{e}^{-w_{j}}$ 's.

Theorem 4.1. Define $\mathcal{L}_{g, n}\left(t_{1}, \ldots, t_{n}\right)$ by

$$
\begin{aligned}
& \mathcal{L}_{g, n}\left(t_{1}, \ldots, t_{n}\right) d t_{1} \otimes \cdots \otimes d t_{n} \\
& \quad=\left(d_{1} \otimes \cdots \otimes d_{n}\right) L_{g, n}\left(w_{1}(t), \ldots, w_{n}(t)\right) \\
& \quad=\frac{\partial^{n}}{\partial w_{1} \cdots \partial w_{n}} L_{g, n}\left(w_{1}, \ldots, w_{n}\right) d w_{1} \otimes \cdots \otimes d w_{n}
\end{aligned}
$$

using the coordinate change (4.2). The differentials $d t_{j}$ and $d w_{j}$ are related by

$$
d w_{j}=\frac{2}{t_{j}^{2}-1} d t_{j}
$$

Then every $\mathcal{L}_{g, n}\left(t_{1}, \ldots, t_{n}\right)$ for $2 g-2+n>0$ is a Laurent polynomial of degree $3 g-3+n$ in $t_{1}^{2}, t_{2}^{2}, \ldots, t_{n}^{2}$. The initial values are

$$
\mathcal{L}_{0,3}\left(t_{1}, t_{2}, t_{3}\right)=-\frac{1}{16}\left(1-\frac{1}{t_{1}^{2} t_{2}^{2} t_{3}^{2}}\right)
$$

and

$$
\mathcal{L}_{1,1}(t)=-\frac{1}{128} \frac{\left(t^{2}-1\right)^{3}}{t^{4}} .
$$

The functions $\mathcal{L}_{g, n}\left(t_{1}, \ldots, t_{n}\right)$ for all $(g, n)$ subject to $2 g-2+n>0$ are uniquely determined by the topological recursion formula

$$
\begin{aligned}
\mathcal{L}_{g, n}\left(t_{N}\right)= & -\frac{1}{16} \sum_{j=2}^{n} \frac{\partial}{\partial t_{j}}\left[\frac { t _ { j } } { t _ { 1 } ^ { 2 } - t _ { j } ^ { 2 } } \left(\frac{\left(t_{1}^{2}-1\right)^{3}}{t_{1}^{2}} \mathcal{L}_{g, n-1}\left(t_{N \backslash\{j\}}\right)\right.\right. \\
& \left.\left.-\frac{\left(t_{j}^{2}-1\right)^{3}}{t_{j}^{2}} \mathcal{L}_{g, n-1}\left(t_{N \backslash\{1\}}\right)\right)\right]
\end{aligned}
$$




$$
\begin{aligned}
& -\frac{1}{32} \frac{\left(t_{1}^{2}-1\right)^{3}}{t_{1}^{2}}\left[\mathcal{L}_{g-1, n+1}\left(t_{1}, t_{1}, t_{N \backslash\{1\}}\right)\right. \\
& \left.+\sum_{\substack{g_{1}+g_{2}=g \\
I \sqcup J=N \backslash\{1\}}}^{\text {stable }} \mathcal{L}_{g_{1},|I|+1}\left(t_{1}, t_{I}\right) \mathcal{L}_{g_{2},|J|+1}\left(t_{1}, t_{J}\right)\right] .
\end{aligned}
$$

Here we use the same convention of notations as in Theorem 3.1.

If we assume (4.4) to (4.6), then it is obvious that $\mathcal{L}_{g, n}\left(t_{N}\right)$ is a Laurent polynomial in $t_{1}^{2}, \ldots, t_{n}^{2}$ of degree $3 g-3+n$. The proof of (4.6) is given in 7 . The initial values (4.4) and (4.5) are calculated in 7.

\section{The Euclidean volume of the moduli space}

In this section we extract the information on the Euclidean volume function from the Laurent polynomial $\mathcal{L}_{g, n}\left(t_{N}\right)$. We then derive a topological recursion for the Laplace transform of the Euclidean volume. Let us recall the Euclidean volume function $v_{g, n}^{\mathrm{E}}(\mathbf{p})$ of $(2.6)$.

Proposition 5.1. Let $V_{g, n}^{\mathrm{E}}\left(t_{N}\right)$ be the homogeneous leading terms of $\mathcal{L}_{g, n}\left(t_{N}\right)$ for $(g, n)$ subject to $2 g-2+n>0$. Then we have

$$
V_{g, n}^{\mathrm{E}}\left(t_{N}\right) d t_{1} \otimes \cdots \otimes d t_{n}=d_{1} \otimes \cdots \otimes d_{n} \int_{\mathbb{R}_{+}^{n}} v_{g, n}^{\mathrm{E}}(\mathbf{p}) \mathrm{e}^{-\langle w, \mathbf{p}\rangle} d p_{1} \cdots d p_{n}
$$

where we change the $w$-variables to the t-variables according to the transformation (4.2).

Proof. From (3.2), we have

$$
\begin{aligned}
\int_{\mathbb{R}_{+}^{n}} v_{g, n}^{\mathrm{E}}(\mathbf{p}) \mathrm{e}^{-\langle w, \mathbf{p}\rangle} d p_{1} \cdots d p_{n} & =\lim _{k \rightarrow \infty} \sum_{\mathbf{p} \in \frac{1}{k} \mathbb{Z}_{+}^{n}} N_{g, n}(k \mathbf{p}) \mathrm{e}^{-\langle w, \mathbf{p}\rangle} \frac{1}{k^{3(2 g-2+n)}} \\
& =\lim _{k \rightarrow \infty} \sum_{\mathbf{p} \in \mathbb{Z}_{+}^{n}} N_{g, n}(\mathbf{p}) \mathrm{e}^{-\frac{1}{k}\langle w, \mathbf{p}\rangle} \frac{1}{k^{3(2 g-2+n)}} \\
& =\lim _{k \rightarrow \infty} L_{g, n}\left(\frac{w_{1}}{k}, \cdots, \frac{w_{n}}{k}\right) \frac{1}{k^{3(2 g-2+n)}}
\end{aligned}
$$


The coordinate transformation (4.2) has the expansion near $w=0$

$$
\begin{aligned}
t & =t(w)=-\frac{2}{w}-\frac{w}{6}+\frac{w^{3}}{360}-\frac{w^{5}}{15,120}+\cdots \\
w & =w(t)=-\frac{2}{t}-\frac{2}{3 t^{3}}-\frac{2}{5 t^{5}}-\cdots
\end{aligned}
$$

Since

$$
\mathcal{L}_{g, n}\left(t_{N}\right)=\frac{\partial^{n}}{\partial t_{1} \cdots \partial t_{n}} L_{g, n}\left(w\left(t_{1}\right), \ldots, w\left(t_{n}\right)\right)
$$

is a Laurent polynomial of degree $2(3 g-3+n)$, and since the change $w \mapsto$ $w / k$ makes

$$
t \longmapsto k t+\mathcal{O}\left(\frac{1}{k}\right)
$$

for a fixed value $t$, we have

$$
\begin{aligned}
& \frac{\partial^{n}}{\partial t_{1} \cdots \partial t_{n}} \int_{\mathbb{R}_{+}^{n}} v_{g, n}^{\mathrm{E}}(\mathbf{p}) \mathrm{e}^{-\langle w(t), \mathbf{p}\rangle} d p_{1} \cdots d p_{n} \\
& \quad=\lim _{k \rightarrow \infty} \frac{\partial^{n}}{\partial t_{1} \cdots \partial t_{n}} L_{g, n}\left(\frac{w\left(t_{1}\right)}{k}, \cdots, \frac{w\left(t_{n}\right)}{k}\right) \frac{1}{k^{3(2 g-2+n)}} \\
& \quad=\lim _{k \rightarrow \infty} \mathcal{L}_{g, n}\left(k t_{1}+\mathcal{O}\left(\frac{1}{k}\right), \cdots, k t_{n}+\mathcal{O}\left(\frac{1}{k}\right)\right) \frac{k^{n}}{k^{3(2 g-2+n)}} \\
& \quad=V_{g, n}^{\mathrm{E}}\left(t_{N}\right) .
\end{aligned}
$$

This completes the proof.

Since $v_{g, n}^{\mathrm{E}}(\mathbf{p})$ is defined by the push-forward measure of the incidence matrix $A_{\Gamma}$ of $(2.2)$ at each point $\Gamma \in R G_{g, n}(\mathbf{p})$, we have

$$
\begin{aligned}
\int_{\mathbb{R}_{+}^{n}} & v_{g, n}^{\mathrm{E}}(\mathbf{p}) \mathrm{e}^{-\langle w, \mathbf{p}\rangle} d p_{1} \cdots d p_{n} \\
= & \sum_{\substack{\Gamma \text { trivalent ribbon } \\
\text { graph of type }(g, n)}} \frac{1}{|\operatorname{Aut}(\Gamma)|} \int_{\mathbb{R}_{+}^{n}} \operatorname{vol}\left(P_{\Gamma}(\mathbf{p})\right) \mathrm{e}^{-\langle w, \mathbf{p}\rangle} d p_{1} \cdots d p_{n} \\
= & \sum_{\substack{\Gamma \text { trivalent ribbon } \\
\text { graph of type }(g, n)}} \frac{1}{|\operatorname{Aut}(\Gamma)|} \int_{\mathbb{R}_{+}^{e(\Gamma)}} \mathrm{e}^{-\left\langle w, A_{\Gamma} \mathbf{x}\right\rangle} d x_{1} \cdots d x_{e(\Gamma)} \\
= & \sum_{\substack{\Gamma \text { trivalent ribbon } \\
\text { graph of type }(g, n)}} \frac{1}{|\operatorname{Aut}(\Gamma)|} \prod_{\eta=1}^{e(\Gamma)} \frac{1}{\left\langle w, a_{\eta}\right\rangle},
\end{aligned}
$$


where $a_{1}, \ldots, a_{e(\Gamma)}$ are columns of the edge-face incidence matrix

$$
A_{\Gamma}=\left[a_{1}\left|a_{2}\right| \cdots \mid a_{e(\Gamma)}\right]
$$

We note that $e(\Gamma)$ takes its maximum value $3(2 g-2+n)$ for a trivalent graph. Thus the last line of (5.3) has a pole of order $3(2 g-2+n)$ at $w=$ 0 . This expression also shows that the leading terms of $L_{g, n}\left(w\left(t_{N}\right)\right)$ as a function in $t_{N}$ using the expansion (5.2) around $t_{N} \sim \infty$ are the Laplace transform of the Euclidean volume function. In particular, we deduce that $N_{g, n}(\mathbf{p})$ behaves asymptotically like a polynomial of degree $2(3 g-3+n)$ for large $\mathbf{p} \in \mathbb{R}_{+}^{n}$.

Since $V_{g, n}^{\mathrm{E}}\left(t_{N}\right)$ is the leading terms of $\mathcal{L}_{g, n}\left(t_{N}\right)$, it is easy to obtain a topological recursion.

Theorem 5.1. The Laplace transformed Euclidean volume function $V_{g, n}^{\mathrm{E}}\left(t_{N}\right)$ in the stable range $2 g-2+n>0$ satisfies the following topological recursion:

$$
\begin{aligned}
V_{g, n}^{\mathrm{E}}\left(t_{N}\right)= & -\frac{1}{16} \sum_{j=2}^{n} \frac{\partial}{\partial t_{j}}\left[\frac{t_{j}}{t_{1}^{2}-t_{j}^{2}}\left(t_{1}^{4} V_{g, n-1}^{\mathrm{E}}\left(t_{N \backslash\{j\}}\right)-t_{j}^{4} V_{g, n-1}^{\mathrm{E}}\left(t_{N \backslash\{1\}}\right)\right)\right] \\
& -\frac{1}{32} t_{1}^{4}\left[V_{g-1, n+1}^{\mathrm{E}}\left(t_{1}, t_{1}, t_{N \backslash\{1\}}\right)\right. \\
& \left.+\sum_{\substack{g_{1}+g_{2}=g \\
I \sqcup J=N \backslash\{1\}}}^{\text {stable }} V_{g_{1},|I|+1}^{\mathrm{E}}\left(t_{1}, t_{I}\right) V_{g_{2},|J|+1}^{\mathrm{E}}\left(t_{1}, t_{J}\right)\right] .
\end{aligned}
$$

Proof. The leading contribution of (4.6) comes from the leading term of

$$
\frac{\left(t^{2}-1\right)^{3}}{t^{2}}=t^{4}-3 t^{2}+3-\frac{1}{t^{2}} .
$$

Thus (4.6) reduces to (5.4).

\section{The symplectic volume of the moduli space and the Kontsevich constants}

Suppose the $i$ th face of a metric ribbon graph $\Gamma \in R G_{g, n}(\mathbf{p})$ consists of edges labeled by $1,2, \ldots, k$ in this cyclic order. (Here again we are abusing the notation to indicate a metric ribbon graph by the same letter $\Gamma$.) If an 


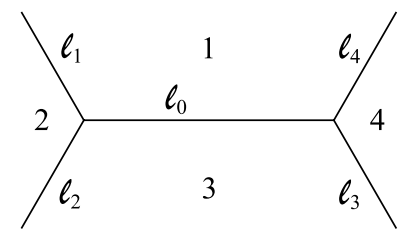

Figure 7: The vector field $X_{0}$.

edge appears twice in this list, then we count it repetitively. Denote by $\ell_{\alpha}$ the length of edge $\alpha$. They satisfy the relation $\ell_{1}+\cdots+\ell_{k}=p_{i}$. Note that the collection of edge lengths forms an orbifold coordinate system on $R G_{g, n}$ at each point $\Gamma$. Kontsevich [30] defines a two-form on $R G_{g, n}$ by

$$
\omega_{K}(\mathbf{p})=\sum_{i=1}^{n} p_{i}^{2} \omega_{i}, \quad \omega_{i}=\sum_{\alpha<\beta} d\left(\frac{\ell_{\alpha}}{p_{i}}\right) \wedge d\left(\frac{\ell_{\beta}}{p_{i}}\right) \quad \text { on face } i
$$

If we change the cyclic order from $(1,2, \ldots, k)$ to $(2,3, \ldots, k, 1)$ and define the form $\omega_{i}^{\prime}$ in the same manner, then we have

$$
\omega_{i}-\omega_{i}^{\prime}=2 d\left(\frac{\ell_{1}}{p_{i}}\right) \wedge d\left(\frac{\ell_{2}+\cdots+\ell_{k}}{p_{i}}\right)=0 .
$$

Therefore, each $\omega_{i}$ and $\omega_{K}(\mathbf{p})$ are well defined as genuine two-forms on $R G_{g, n}$. The restriction of the two-form $\omega_{K}(\mathbf{p})$ defines a symplectic structure on $R G_{g, n}(\mathbf{p}) \cong \mathcal{M}_{g, n}$ for each $\mathbf{p} \in \mathbb{R}_{+}^{n}$.

To see the non-degeneracy of $\omega_{K}(\mathbf{p})$, let us analyze the perimeter map $\pi$ locally around a trivalent ribbon graph $\Gamma$. As in Section 2 we give a name to all edges of $\Gamma$, this time without repetition, indexed by $\{0,1,2, \ldots, e(\Gamma)-1\}$. Faces of $\Gamma$ are indexed by $N=\{1,2, \ldots, n\}$. The edge-face incidence matrix $A_{\Gamma}$ of (2.2) gives the differential of the perimeter map

$$
A_{\Gamma}=d \pi_{\Gamma}
$$

at the metric ribbon graph $\Gamma$ if it is trivalent. To set notations simple, we assume that faces 1-4 and edges $0-4$ are arranged as in figure 7 .

Define the vector field

$$
X_{0}=-\frac{\partial}{\partial \ell_{1}}+\frac{\partial}{\partial \ell_{2}}-\frac{\partial}{\partial \ell_{3}}+\frac{\partial}{\partial \ell_{4}} .
$$


We then have

$$
\begin{aligned}
& \left.\iota_{X_{0}}\left(p_{1}^{2} \omega_{1}\right)\right|_{R G_{g, n}(\mathbf{p})}=-d \ell_{0}-d \ell_{1}-d \ell_{4}, \\
& \left.\iota_{X_{0}}\left(p_{2}^{2} \omega_{2}\right)\right|_{R G_{g, n}(\mathbf{p})}=d \ell_{1}+d \ell_{2}, \\
& \left.\iota_{X_{0}}\left(p_{3}^{2} \omega_{3}\right)\right|_{R G_{g, n}(\mathbf{p})}=-d \ell_{0}-d \ell_{2}-d \ell_{3}, \\
& \left.\iota_{X_{0}}\left(p_{4}^{2} \omega_{4}\right)\right|_{R G_{g, n}(\mathbf{p})}=d \ell_{3}+d \ell_{4} .
\end{aligned}
$$

Therefore,

$$
\left.\iota_{X_{0}} \omega_{K}(\mathbf{p})\right|_{R G_{g, n}(\mathbf{p})}=-2 d \ell_{0}
$$

on the tangent space $T_{\Gamma} R G_{g, n}(\mathbf{p})$. This shows that the two-form $\omega_{K}(\mathbf{p})$ restricted on $\operatorname{Ker}\left(d \pi_{\Gamma}\right)$ is a linear isomorphism. We refer to [2] for more detail.

Alternatively, we can introduce the symplectic structure on $R G_{r, n}(\mathbf{p})$ through symplectic reduction. The ribbon graph complex $R G_{g, n}$ comes with a natural fibration on it, the tautological torus bundle

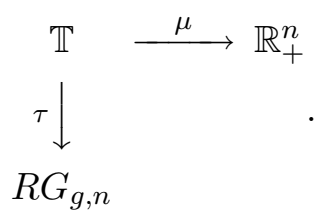

The fiber of $\tau$ at a metric ribbon graph $\Gamma$ is the cartesian product of the boundary of the $n$ faces of $\Gamma$, which is identified with the collection of $n$ polygons. Topologically each fiber of $\mathbb{T}$ is an $n$-dimensional torus $T^{n}=\left(S^{1}\right)^{n}$. We use the same letter $\mathbb{T}$ for the total space of this torus bundle, whose dimension $2(3 g-3+2 n)$ is always even.

The identification of the $i$ th face of $\Gamma \in R G_{r, n}(\mathbf{p})$ and the circle $S^{1}=$ $\mathbb{R} / \mathbb{Z}$ is given as follows. First we choose a vertex on the $i$ th polygon, and name the edges on the $i$ th face as $1,2, \ldots, k$ in this cyclic order such that the chosen vertex is the beginning point of edge 1 and the end point of edge $k$. Let $\ell_{\alpha}$ be the length of edge $\alpha$ as before. We choose a parameter $\phi_{i}$ subject to $0 \leq \phi_{i} \leq \ell_{1}$. Under the re-naming of the edges $(1,2, \ldots, k) \longmapsto$ $(2,3, \ldots, k, 1), \phi_{i}$ changes to $\phi_{i}^{\prime}=\phi_{i}+\ell_{1}$. The choice of the vertex and $\phi_{i}$ is identified with an element of $S^{1}$, and also determines the torus action on the fibration $\mathbb{T}$. 
Define a two-form $\Omega$ by

$$
\begin{aligned}
\Omega & =\sum_{i=1}^{n} \hat{\omega}_{i}, \\
\hat{\omega}_{i} & =\sum_{\alpha<\beta} d \ell_{\alpha} \wedge d \ell_{\beta}+d\left(\frac{\phi_{i}}{p_{i}}\right) \wedge d\left(p_{i}^{2}\right),
\end{aligned}
$$

The cyclic re-naming of edges changes $\hat{\omega}_{i}$ to

$$
\hat{\omega}_{i}^{\prime}=\sum_{2 \leq \alpha<\beta} d \ell_{\alpha} \wedge d \ell_{\beta}+\sum_{2 \leq \alpha} d \ell_{\alpha} \wedge d \ell_{1}+d\left(\frac{\phi_{i}+\ell_{1}}{p_{i}}\right) \wedge d\left(p_{i}^{2}\right)
$$

Therefore,

$$
\hat{\omega}_{i}-\hat{\omega}_{i}^{\prime}=\sum_{2 \leq \beta} d \ell_{1} \wedge d \ell_{\beta}-\sum_{2 \leq \alpha} d \ell_{\alpha} \wedge d \ell_{1}+2 d \ell_{1} \wedge d p_{i}=0
$$

and hence $\Omega$ is a globally well-defined two-form on the total space $\mathbb{T}$. The moment map of the torus action on $\mathbb{T}$ is the assignment

$$
\mu: \mathbb{T} \ni\left(\Gamma, \phi_{1}, \ldots, \phi_{n}\right) \longmapsto\left(p_{1}^{2}, \ldots, p_{n}^{2}\right) \in \mathbb{R}_{+}^{n}
$$

The symplectic quotient $\mu^{-1}(L) / / T^{n}$ of $\mathbb{T}$ by this torus action is $\left(R G_{g, n}(\mathbf{p})\right.$, $\left.\omega_{K}(\mathbf{p})\right)$ of $(6.1)$.

Now we define the symplectic volume of the moduli space $\mathcal{M}_{g, n} \cong$ $R G_{g, n}(\mathbf{p})$ by

$$
v_{g, n}^{\mathrm{S}}(\mathbf{p})=\int_{R G_{g, n}(\mathbf{p})} \exp \left(\omega_{K}(\mathbf{p})\right)
$$

Applying the recursion argument similar to our proof of Theorem 3.1 to the symplectic reduction of $R G_{g, n}$ by the torus action, the following theorem was established in [2]. 
Theorem 6.1 [2]. The symplectic volume satisfies the following topological recursion:

$$
\begin{aligned}
p_{1} v_{g, n}^{\mathrm{S}}\left(p_{N}\right)= & \sum_{j=2}^{n}\left[\int_{0}^{p_{1}+p_{j}} q\left(p_{1}+p_{j}-q\right) v_{g, n-1}^{\mathrm{S}}\left(q, p_{N \backslash\{1, j\}}\right) d q\right. \\
& +H\left(p_{1}-p_{j}\right) \int_{0}^{p_{1}-p_{j}} q\left(p_{1}-p_{j}-q\right) v_{g, n-1}^{\mathrm{S}}\left(q, p_{N \backslash\{1, j\}}\right) d q \\
& \left.-H\left(p_{j}-p_{1}\right) \int_{0}^{p_{j}-p_{1}} q\left(p_{j}-p_{1}-q\right) v_{g, n-1}^{\mathrm{S}}\left(q, p_{N \backslash\{1, j\}}\right) d q\right] \\
& +2 \iint_{0 \leq q_{1}+q_{2} \leq p_{1}} q_{1} q_{2}\left(p_{1}-q_{1}-q_{2}\right)\left[v_{g-1, n+1}^{\mathrm{S}}\left(q_{1}, q_{2}, p_{N \backslash\{1\}}\right)\right. \\
& \left.+\sum_{\substack{g_{1}+g_{2}=g \\
I \sqcup J=N \backslash\{1\}}}^{\operatorname{stable}} v_{g_{1},|I|+1}^{\mathrm{S}}\left(q_{1}, p_{I}\right) v_{g_{2},|J|+1}\left(q_{2}, p_{J}\right) d q_{1} d q_{2}\right] .
\end{aligned}
$$

The initial values are easy to calculate. For the case of $(g, n)=(0,3)$, since the perimeter $\left(p_{1}, p_{2}, p_{3}\right) \in \mathbb{R}_{+}^{3}$ determines the length of each edge, the symplectic form is 1 on a single point. Thus we have

$$
v_{0,3}^{\mathrm{S}}\left(p_{1}, p_{2}, p_{3}\right)=1
$$

The unique trivalent graph of type $(g, n)=(1,1)$ is given in figure 8 , which has the automorphism group $\mathbb{Z} / 6 \mathbb{Z}$. The perimeter map is given by $p=$ $2\left(\ell_{1}+\ell_{2}+\ell_{3}\right)$. The restriction of $\omega_{K}(p)$ on $R G_{1,1}(p)$ is $2 d \ell_{1} \wedge d \ell_{2}$. Therefore, we have

$$
v_{1,1}^{\mathrm{S}}(p)=\frac{1}{6} \int_{0 \leq \ell_{1}+\ell_{2} \leq \frac{p}{2}} 2 d \ell_{1} \wedge d \ell_{2}=\frac{1}{24} p^{2} .
$$

We now consider the Laplace transform of the symplectic volume $v_{g, n}^{\mathrm{S}}(\mathbf{p})$.

Theorem 6.2. The symmetric function $V_{g, n}^{\mathrm{S}}\left(t_{N}\right)$ defined by the Laplace transform

$$
V_{g, n}^{\mathrm{S}}\left(t_{1}, \ldots, t_{n}\right) d t_{1} \otimes \cdots \otimes d t_{n} \stackrel{\text { def }}{=} d_{1} \otimes \cdots \otimes d_{n} \int_{\mathbb{R}_{+}^{n}} v_{g, n}^{\mathrm{S}}(\mathbf{p}) \mathrm{e}^{-\langle w, \mathbf{p}\rangle} d p_{1} \cdots d p_{n}
$$



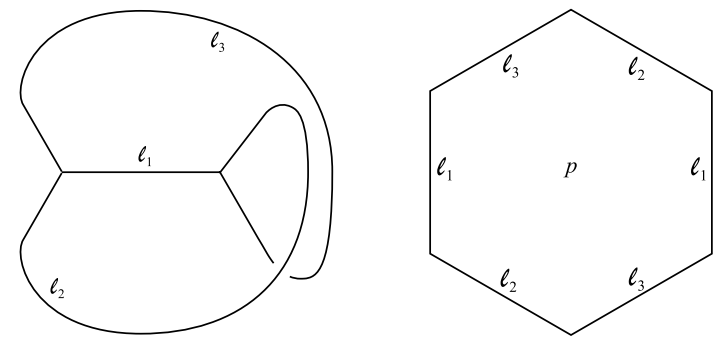

Figure 8: The trivalent ribbon graph of type $(1,1)$.

and the coordinate change

$$
w_{j}=-\frac{2}{t_{j}}
$$

satisfies the topological recursion

$$
\begin{aligned}
V_{g, n}^{\mathrm{S}}\left(t_{N}\right)= & -\frac{1}{4} \sum_{j=2}^{\infty} \frac{\partial}{\partial t_{j}}\left[\frac{t_{j}}{t_{1}^{2}-t_{j}^{2}}\left(t_{1}^{4} V_{g, n-1}^{\mathrm{S}}\left(w_{N \backslash\{j\}}\right)-t_{j}^{4} V_{g, n-1}^{\mathrm{S}}\left(w_{N \backslash\{1\}}\right)\right)\right] \\
& -\frac{1}{4} t_{1}^{4}\left(V_{g-1, n+1}^{\mathrm{S}}\left(t_{1}, t_{1}, t_{N \backslash\{1\}}\right)\right. \\
& \left.+\sum_{\substack{g_{1}+g_{2}=g, I \sqcup J=N \backslash\{1\}}} V_{g_{1},|I|+1}^{\mathrm{S}}\left(t_{1}, t_{I}\right) V_{g_{2},|J|+1}^{\mathrm{S}}\left(t_{1}, t_{J}\right)\right)
\end{aligned}
$$

The proof of this theorem is given in 7 . The very reason that Kontsevich was interested in the symplectic volume of the moduli space is that it gives the generating function of the intersection numbers (1.2)

$$
V_{g, n}^{\mathrm{S}}\left(t_{N}\right)=(-1)^{n} \sum_{\substack{d_{1}+\cdots d_{n} \\=3 g-3+n}}\left\langle\tau_{d_{1}} \cdots \tau_{d_{n}}\right\rangle_{g, n} \prod_{j=1}^{n}\left(2 d_{j}+1\right) ! !\left(\frac{t_{j}}{2}\right)^{2 d_{j}}
$$

The topological recursion (6.11) produces a relation among the coefficients, which is known as the DVV formula of [9], and is equivalent to the Virasoro constraint condition of [51]. 
Since the volume is for $\mathcal{M}_{g, n}$ and the intersection numbers are for $\overline{\mathcal{M}}_{g, n}$, it is not obvious why they are the same thing. From the deep theory of Mirzakhani [34,35], it becomes obvious why and how they are related.

We are now ready to calculate the Kontsevich constants.

Theorem 6.3. The ratio of the two volume polynomials $V_{g, n}^{\mathrm{S}}\left(t_{N}\right)$ and $V_{g, n}^{\mathrm{E}}\left(t_{N}\right)$ is a constant depending only on $g$ and $n$ :

$$
\rho_{g, n}(t) \stackrel{\text { def }}{=} \frac{V_{g, n}^{\mathrm{S}}\left(t_{N}\right)}{V_{g, n}^{\mathrm{E}}\left(t_{N}\right)}=2^{5 g-5+2 n}
$$

Proof. We use induction on $2 g-2+n$. From (6.7), (6.8), (B.3) and (B.6), we have

$$
\left\{\begin{array} { l } 
{ V _ { 0 , 3 } ^ { \mathrm { E } } ( t _ { 1 } , t _ { 2 } , t _ { 3 } ) = - \frac { 1 } { 1 6 } , } \\
{ V _ { 0 , 3 } ^ { \mathrm { S } } ( t _ { 1 } , t _ { 2 } , t _ { 3 } ) = - \frac { 1 } { 8 } , }
\end{array} \quad \text { and } \quad \left\{\begin{array}{l}
V_{1,1}^{\mathrm{E}}(t)=-\frac{1}{128} t^{2} \\
V_{1,1}^{\mathrm{S}}(t)=-\frac{1}{32} t^{2}
\end{array}\right.\right.
$$

Thus the initial values satisfy (6.13). We observe that the recursion formulas (5.4) and (6.11) are the same except for the constant factors on the first and the second lines of the right-hand side. Therefore, if we changed $V_{g, n}^{\mathrm{E}}\left(t_{N}\right)$ to $2^{5 g-5+2 n} \cdot V_{g, n}^{\mathrm{E}}\left(t_{N}\right)$ in (5.4), then its recursion formula would become identical to (6.11). Since the recursion uniquely determines all values for $(g, n)$ subject to $2 g-2+n>0$ from the initial values (6.14), we establish (6.13). This completes the proof.

\section{The Eynard-Orantin theory on $\mathbb{P}^{1}$}

The number of integral ribbon graphs $N_{g, n}(\mathbf{p})$ is a difficult function to deal with because it is not given by a single formula. As we have noted, it behaves like a polynomial for large $\mathbf{p} \in \mathbb{Z}_{+}^{n}$, while it takes value 0 whenever $p_{1}+$ $\cdots+p_{n}$ is odd. Compared to this, the Laplace transformed function such as $\mathcal{L}_{g, n}\left(t_{N}\right)$ is a far nicer object. Indeed $\mathcal{L}_{g, n}\left(t_{N}\right)$ is a Laurent polynomial and satisfies a simple differential recursion formula (4.6). We also note that the recursion formulas (4.6), (5.4) and (6.11) take a very similar shape. Over the years several authors (including $[4,5,8,14-17,30,33,38,45,53,54]$ ) have noticed that many different combinatorial structures (on the A-model side of a topological string theory) can be uniformly treated on the B-model side, 
after taking the Laplace transform. The importance of the Laplace transform as the mirror map was noted in [15]. This uniform structure after the Laplace transform is the manifestation of the Eynard-Orantin theory. We will show in this section that the recursions (4.6), (5.4), and (6.11) become identical under the formalism proposed in [16].

We are not in the place to formally present the Eynard-Orantin formalism in an axiomatic way. Instead of giving the full account, we are satisfied with explaining a limited case when the spectral curve of the theory is $\mathbb{P}^{1}$. The word "spectral curve" was used in [16] because of the analogy of the spectral curves appearing in the Lax formalism of integrable systems.

We start with the spectral curve $C=\mathbb{P}^{1} \backslash S$, where $S \subset \mathbb{P}^{1}$ is a finite set. We also need two generic elements $x$ and $y$ of $H^{0}\left(C, \mathcal{O}_{C}\right)$, where $\mathcal{O}_{C}$ denotes the sheaf of holomorphic functions on $C$. The condition we impose on $x$ and $y$ is that the holomorphic maps

$$
x: C \longrightarrow \mathbb{C} \text { and } y: C \longrightarrow \mathbb{C}
$$

have only simple ramification points, i.e., their derivatives $d x$ and $d y$ have simple zeros, and that

$$
(x, y): C \ni t \longmapsto(x(t), y(t)) \in \mathbb{C}^{2}
$$

is an immersion. Let $\Lambda^{1}(C)$ denote the sheaf of meromorphic one-forms on $C$, and

$$
H^{n}=H^{0}\left(C^{n}, \operatorname{Sym}^{n}\left(\Lambda^{1}(C)\right)\right)
$$

the space of meromorphic symmetric differentials of degree $n$. The Cauchy differentiation kernel is an example of such differentials:

$$
W_{0,2}\left(t_{1}, t_{2}\right)=\frac{d t_{1} \otimes d t_{2}}{\left(t_{1}-t_{2}\right)^{2}} \in H^{2} .
$$

In the literatures starting from [16], the Cauchy differentiation kernel has been called the Bergman kernel, even thought it has nothing to do with the Bergman kernel in complex analysis. A bilinear operator

$$
K: H \otimes H \longrightarrow H
$$


naturally extends to

$$
\begin{aligned}
K & : H^{n_{1}+1} \otimes H^{n_{2}+1} \ni\left(f_{0}, f_{1}, \ldots, f_{n_{1}}\right) \otimes\left(h_{0}, h_{1}, \ldots, h_{n_{2}}\right) \\
& \longmapsto\left(K\left(f_{0}, h_{0}\right), f_{1}, \ldots, f_{n_{1}}, h_{1}, \ldots, h_{n_{2}}\right) \in H^{n_{1}+n_{2}+1} \\
K & : H^{n+1} \ni\left(f_{0}, f_{1}, \ldots, f_{n_{1}}\right) \longmapsto\left(K\left(f_{0}, f_{1}\right), f_{2}, \ldots, f_{n_{1}}\right) \in H^{n} .
\end{aligned}
$$

Suppose we are given an infinite sequence $\left\{W_{g, n}\right\}$ of differentials $W_{g, n} \in H^{n}$ for all $(g, n)$ subject to the stability condition $2 g-2+n>0$. We say this sequence satisfies a topological recursion with respect to the kernel $K$ if

$$
\begin{aligned}
W_{g, n}= & K\left(W_{g, n-1}, W_{0,2}\right)+K\left(W_{g-1, n+1}\right) \\
& +\frac{1}{2} \sum_{\substack{g_{1}+g_{2}=g \\
I \sqcup J=N \backslash\{1\}}}^{\text {stable }} K\left(W_{g_{1},|I|+1}, W_{g_{2},|J|+1}\right) .
\end{aligned}
$$

The characteristic of the Eynard-Orantin theory lies in the particular choice of the Eynard kernel that reflects the parametrization (7.2) and the ramified coverings (7.1). Let $\mathcal{A}=\left\{a_{1}, \ldots, a_{r}\right\} \subset C$ be the set of simple ramification points of the $x$-projection map. Since locally at each $a_{\lambda}$ the $x$-projection is a double-sheeted covering, we can choose the deck transformation map

$$
s_{\lambda}: U_{\lambda} \stackrel{\sim}{\longrightarrow} U_{\lambda}
$$

where $U_{\lambda} \subset C$ is an appropriately chosen simply connected neighborhood of $a_{\lambda}$.

Definition 7.1. The Eynard kernel is the linear map $H \otimes H \rightarrow H$ defined by

$$
\begin{aligned}
& K\left(f_{1}\left(t_{1}\right) d t_{1}, f_{2}\left(t_{2}\right) d t_{2}\right) \\
& =\frac{1}{2 \pi \mathrm{i}} \sum_{\lambda=1}^{r} \oint_{\left|t-a_{\lambda}\right|<\epsilon} K_{\lambda}\left(t, t_{1}\right)\left(f_{1}(t) d t \otimes f_{2}\left(s_{\lambda}(t)\right) d s_{\lambda}(t)\right. \\
& \left.\quad+f_{2}(t) d t \otimes f_{1}\left(s_{\lambda}(t)\right) d s_{\lambda}(t)\right),
\end{aligned}
$$

where

$$
K_{\lambda}\left(t, t_{1}\right)=\frac{1}{2}\left(\int_{t}^{s_{\lambda}(t)} W_{0,2}\left(t, t_{1}\right) d t\right) \otimes d t_{1} \frac{1}{\left(y(t)-y\left(s_{\lambda}(t)\right)\right) d x(t)}
$$


and $\frac{1}{d x(t)}$ is the contraction operator with respect to the vector field

$$
\left(\frac{d x}{d t}\right)^{-1} \frac{\partial}{\partial t}
$$

The integration is taken with respect to the $t$-variable along a small loop around $a_{\lambda}$ that contains no singularities other than $t=a_{\lambda}$. A topological recursion with respect to the Eynard kernel is what we call the EynardOrantin recursion in this paper.

To convert (4.6) to the Eynard-Orantin formalism, we need to identify the spectral curve of the theory and the unstable case $\mathcal{L}_{0,2}\left(t_{1}, t_{2}\right)$. The spectral curve is a plane algebraic curve

$$
C=\left\{(x, y) \in \mathbb{C}^{2} \mid x y=y^{2}+1\right\},
$$

which is the same curve considered in [41]. Here we introduce a different parametrization

$$
\begin{aligned}
& x(t)=\frac{t+1}{t-1}+\frac{t-1}{t+1}=2+\frac{4}{t^{2}-1}, \\
& y(t)=\frac{t+1}{t-1}
\end{aligned}
$$

with a parameter $t \in \mathbb{P}^{1} \backslash\{1,-1\}$ so that the resulting differentials become Laurent polynomials. This use of the parametrization is similar to that of $[15,38]$. The $x$-projection

$$
\pi: C \ni t \longmapsto x(t) \in \mathbb{C}
$$

has simple ramification points at $t=0$ and $\infty$, since

$$
d x=-\frac{8 t}{\left(t^{2}-1\right)^{2}} d t .
$$

We note that since the map $\pi$ is globally a branched double-sheeted covering, its covering transformation is globally defined and is given by

$$
s: C \ni t \longmapsto s(t)=-t \in C \text {. }
$$

The unstable $(0,2)$ case is calculated in $7,($ B.9). The result is

$$
\mathcal{L}_{0,2}\left(t_{1}, t_{2}\right) d t_{1} \otimes d t_{2}=\frac{d t_{1} \otimes d t_{2}}{\left(t_{1}+t_{2}\right)^{2}}=\frac{d t_{1} \otimes d t_{2}}{\left(t_{1}-s\left(t_{2}\right)\right)^{2}} .
$$


This quadratic differential form plays the role of the Cauchy differentiation kernel. For every holomorphic differential $f(t) d t$ on $C$, we have

$$
\begin{gathered}
-\frac{1}{2 \pi \mathrm{i}} \oint \frac{1}{d t}\left[f(s(t)) d s(t) \mathcal{L}_{0,2}\left(t, t_{1}\right) d t \otimes d t_{1}+f(t) d t \mathcal{L}_{0,2}\left(s(t), t_{1}\right) d s(t) \otimes d t_{1}\right] \\
=\left(\frac{1}{2 \pi \mathrm{i}} \oint \frac{f(-t)}{\left(t+t_{1}\right)^{2}} d t\right) \otimes d t_{1}+\left(\frac{1}{2 \pi \mathrm{i}} \oint \frac{f(t)}{\left(t-t_{1}\right)^{2}} d t\right) \otimes d t_{1}=2 f^{\prime}\left(t_{1}\right) d t_{1}
\end{gathered}
$$

where the operation $\frac{1}{d t}$ is the contraction by the vector field $\frac{\partial}{\partial t}$, and the integration is taken with respect to $t$ along a positively oriented simple loop that contains both $t_{1}$ and $s\left(t_{1}\right)$. Actually, the contour integral should be considered as the residue calculation at $t=\infty$ with respect to the opposite orientation. This explains the minus sign in (7.14).

Theorem 7.1. The topological recursion (4.6) is equivalent to the EynardOrantin recursion of [16]:

$$
\begin{aligned}
\mathcal{L}_{g, n}\left(t_{N}\right) d t_{N} \\
=\frac{1}{2 \pi \mathrm{i}} \int_{\Gamma} K\left(t, t_{1}\right)\left[\sum _ { j = 2 } ^ { n } \left(\mathcal{L}_{g, n-1}\left(t, t_{N \backslash\{1, j\}}\right) d t\right.\right. \\
\quad \otimes d t_{N \backslash\{1, j\}} \otimes \mathcal{L}_{0,2}\left(s(t), t_{j}\right) d s(t) \otimes d t_{j} \\
\left.\quad+\mathcal{L}_{g, n-1}\left(s(t), t_{N \backslash\{1, j\}}\right) d s(t) \otimes d t_{N \backslash\{1, j\}} \otimes \mathcal{L}_{0,2}\left(t, t_{j}\right) d t \otimes d t_{j}\right) \\
\quad+\mathcal{L}_{g-1, n+1}\left(t, s(t), t_{N \backslash\{1\}}\right) d t \otimes d s(t) \otimes d t_{N \backslash\{1\}} \\
\quad+\sum_{\substack{g_{1}+g_{2}=g \\
I \sqcup J=N \backslash\{1\}}}^{\text {stable }}\left(\mathcal{L}_{g_{1},|I|+1}\left(t, t_{I}\right) d t \otimes d t_{I}\right) \\
\left.\quad \otimes\left(\mathcal{L}_{g_{2},|J|+1}\left(s(t), t_{J}\right) d s(t) \otimes d t_{J}\right)\right] .
\end{aligned}
$$

Here the contour integration is taken with respect to $t$ along a curve $\Gamma$ that consists of a large circle of the negative orientation centered at the origin with radius $r>\max _{j \in N}\left|t_{j}\right|$, and a small circle around the origin of the positive orientation. We use a simplified notation $d t_{I}=\bigotimes_{i \in I} d t_{i}$ for $I \subset N$.

Remark 7.1. 1. The contour integral (7.15) can be phrased as the sum of the residues of the integrand at the raminfication points of the spectral curve $t=0$ and $\infty$, which is the language used in [16]. 
2. The first and the second lines of the right-hand side of (7.15) are unstable $(0,2)$ cases of the fourth line when we have $\left(g_{1}, I\right)=(0,\{j\})$ or $\left(g_{2}, J\right)=(0,\{j\})$.

3. In terms of the Cauchy differentiation kernel $W_{0,2}\left(t, t_{1}\right)$ of $(7.4)$, we have

$$
\mathcal{L}_{0,2}\left(t, t_{1}\right) d t \otimes d t_{1}=W_{0,2}\left(t, t_{1}\right)-\pi^{*} W_{0,2}\left(x, x_{1}\right)
$$

as proved in $7,(\mathrm{~B} .10)$. Since $\pi^{*} W_{0,2}\left(x, x_{1}\right)$ is invariant under the deck transformation $s: C \rightarrow C$ applied to the entry $x$, and since $\mathcal{L}_{g, n}\left(t_{N}\right)$ is an even function by Theorem 4.1 , we can replace $\mathcal{L}_{0,2}\left(t, t_{1}\right) d t \otimes d t_{1}$ with $W_{0,2}\left(t, t_{1}\right)$ in $(7.15)$.

Proof. The Eynard kernel of our setting is

$$
\begin{aligned}
K\left(t, t_{1}\right) & =\frac{1}{2}\left(\int_{t}^{s(t)} \mathcal{L}_{0,2}\left(t, t_{1}\right) d t\right) \otimes d t_{1} \frac{1}{(y(t)-y(s(t))) d x(t)} \\
& =\frac{1}{2}\left(\int_{t}^{s(t)} \frac{d t}{\left(t+t_{1}\right)^{2}}\right) \otimes d t_{1} \frac{1}{\left(\frac{t+1}{t-1}-\frac{-t+1}{-t-1}\right) \frac{\partial}{\partial t}\left(\frac{t+1}{t-1}+\frac{t-1}{t+1}\right)} \frac{1}{d t} \\
& =-\frac{1}{2}\left(\frac{1}{t-t_{1}}+\frac{1}{t+t_{1}}\right) \frac{1}{32} \frac{\left(t^{2}-1\right)^{3}}{t^{2}} \frac{1}{d t} \otimes d t_{1} .
\end{aligned}
$$

Thus for any symmetric Laurent polynomial $f(t, s)$ in $t^{2}$ and $s^{2}$, we have

$$
\frac{1}{2 \pi \mathrm{i}} \int_{\Gamma} K\left(t, t_{1}\right) f(t, s(t)) d t \otimes d s(t)=-f\left(t_{1}, t_{1}\right) \frac{1}{32} \frac{\left(t_{1}^{2}-1\right)^{3}}{t_{1}^{2}} d t_{1}
$$

since $s(t)=-t$. Therefore, the third and the fourth lines of the right-hand side of (7.15) become

$$
-\frac{1}{32} \frac{\left(t_{1}^{2}-1\right)^{3}}{t_{1}^{2}}\left[\mathcal{L}_{g-1, n+1}\left(t_{1}, t_{1}, t_{N \backslash\{1\}}\right)\right.
$$




$$
\left.+\sum_{\substack{g_{1}+g_{2}=g \\ I \sqcup J=N \backslash\{1\}}}^{\text {stable }} \mathcal{L}_{g_{1},|I|+1}\left(t_{1}, t_{I}\right) \mathcal{L}_{g_{2},|J|+1}\left(t_{1}, t_{J}\right)\right] \otimes d t_{N} .
$$

This is because $\mathcal{L}_{g, n}\left(t_{N}\right)$ for $(g, n)$ in the stable range is a Laurent polynomial in $t_{1}^{2}, \ldots, t_{n}^{2}$, hence the only simple poles in the complex $t$-plane within the contour $\Gamma$ of (7.15) that appear in the third and fourth lines are located at $t=t_{1}$ and $-t_{1}$.

Even though the first and the second lines of the right-hand side of (7.15) are somewhat a degenerate case of the fourth line as remarked above, the analysis becomes different because $\mathcal{L}_{0,2}\left(t, t_{j}\right)$ contributes new poles in the $t$-plane. First we note that

$$
\begin{aligned}
& \mathcal{L}_{g, n-1}\left(t, t_{N \backslash\{1, j\}}\right) d t \otimes d t_{N \backslash\{1, j\}} \otimes \mathcal{L}_{0,2}\left(s(t), t_{j}\right) d s(t) \otimes d t_{j} \\
& +\mathcal{L}_{g, n-1}\left(s(t), t_{N \backslash\{1, j\}}\right) d s(t) \otimes d t_{N \backslash\{1, j\}} \otimes \mathcal{L}_{0,2}\left(t, t_{j}\right) d t \otimes d t_{j} \\
& \quad=-\mathcal{L}_{g, n-1}\left(t, t_{N \backslash\{1, j\}}\right)\left(\frac{1}{\left(-t+t_{j}\right)^{2}}+\frac{1}{\left(t+t_{j}\right)^{2}}\right) d t^{\otimes 2} \otimes d t_{N \backslash\{1\}} \\
& =-2 \mathcal{L}_{g, n-1}\left(t, t_{N \backslash\{1, j\}}\right) \frac{\partial}{\partial t_{j}} \frac{t_{j}}{t^{2}-t_{j}^{2}} d t^{\otimes 2} \otimes d t_{N \backslash\{1\}}
\end{aligned}
$$

Apply the operation $\frac{1}{2 \pi \mathrm{i}} \int_{\Gamma} K\left(t, t_{1}\right)$ to $(7.16)$ and collect the residues at $t=t_{1}$ and $t_{1}$. We then obtain

$$
-\frac{1}{16} \frac{\partial}{\partial t_{j}}\left[\frac{t_{j}}{t_{1}^{2}-t_{j}^{2}} \frac{\left(t_{1}^{2}-1\right)^{3}}{t_{1}^{2}} \mathcal{L}_{g, n-1}\left(t_{N \backslash\{j\}}\right)\right] d t_{N}
$$

When $t \sim t_{j}$ or $-t_{j}$, we use (7.14) to derive

$$
\begin{aligned}
& -\frac{1}{2 \pi \mathrm{i}} \int_{\Gamma} \frac{t}{t^{2}-t_{1}^{2}} \frac{1}{32} \frac{\left(t^{2}-1\right)^{3}}{t^{2}} \cdot \frac{1}{d t} \otimes d t_{1} \\
& \otimes(-1) \mathcal{L}_{g, n-1}\left(t, t_{N \backslash\{1, j\}}\right)\left(\frac{1}{\left(-t+t_{j}\right)^{2}}+\frac{1}{\left(t+t_{j}\right)^{2}}\right) d t^{\otimes 2} \otimes d t_{N \backslash\{1\}} \\
& \quad=-\frac{1}{32} \frac{\partial}{\partial t_{j}}\left[\frac{t_{j}}{t_{j}^{2}-t_{1}^{2}} \frac{\left(t_{j}^{2}-1\right)^{3}}{t_{j}^{2}} \mathcal{L}_{g, n-1}\left(t_{j}, t_{N \backslash\{1, j\}}\right)\right] d t_{N}
\end{aligned}
$$




$$
\begin{aligned}
& -\frac{1}{32}\left(-\frac{\partial}{\partial t_{j}}\right)\left[\frac{-t_{j}}{t_{j}^{2}-t_{1}^{2}} \frac{\left(t_{j}^{2}-1\right)^{3}}{t_{j}^{2}} \mathcal{L}_{g, n-1}\left(-t_{j}, t_{N \backslash\{1, j\}}\right)\right] d t_{N} \\
& =\frac{1}{16} \frac{\partial}{\partial t_{j}}\left[\frac{t_{j}}{t_{1}^{2}-t_{j}^{2}} \frac{\left(t_{j}^{2}-1\right)^{3}}{t_{j}^{2}} \mathcal{L}_{g, n-1}\left(t_{j}, t_{N \backslash\{1, j\}}\right)\right] d t_{N} .
\end{aligned}
$$

This completes the proof.

Here we note that the spectral curve (7.11), and hence the topological recursion theory of our case, has a non-trivial automorphism. It is given by the transformation

$$
t \longmapsto \frac{1}{t}
$$

which induces an automorphism

$$
C \ni(x, y) \longmapsto(-x,-y) \in C
$$

of the spectral curve. It interchanges the two ramification points of figure 9 . Let

$$
u=\frac{1}{t}, \quad u_{j}=\frac{1}{t_{j}} \quad \text { for } j=1,2, \ldots, n .
$$

Then we have

$$
\mathcal{L}_{0,2}\left(t, t_{j}\right) d t \otimes d t_{j}=\mathcal{L}_{0,2}\left(u, u_{j}\right) d u \otimes d u_{j}
$$

and $y d x=(-y) d(-x)$. It follows that $K\left(t, t_{1}\right)=K\left(u, u_{1}\right)$, and we have $\mathbb{Z} / 2 \mathbb{Z}$ as the automorphism group of the theory. Reflecting this automorphism, the function $\mathcal{L}_{g, n}\left(t_{N}\right)$ exhibits the following transformation property:

$$
\mathcal{L}_{g, n}\left(\frac{1}{t_{1}}, \ldots, \frac{1}{t_{n}}\right)=(-1)^{n} \mathcal{L}_{g, n}\left(t_{1}, \ldots, t_{n}\right) t_{1}^{2} \cdots t_{n}^{2}
$$

The reason that we choose $t$ as our preferred parameter rather than $1 / t$ in (4.2) is to extract the polynomial behavior of the Laplace transform of the Euclidean volume. As $t \rightarrow \infty$ the spectral curve $C$ degenerates to a parabola, and the theory changes from counting the integral ribbon graphs to calculating the Euclidean volume, as we shall see below. By the symmetry argument, the $t \rightarrow 0$ limit also deforms $C$ to a parabola. We can see from 


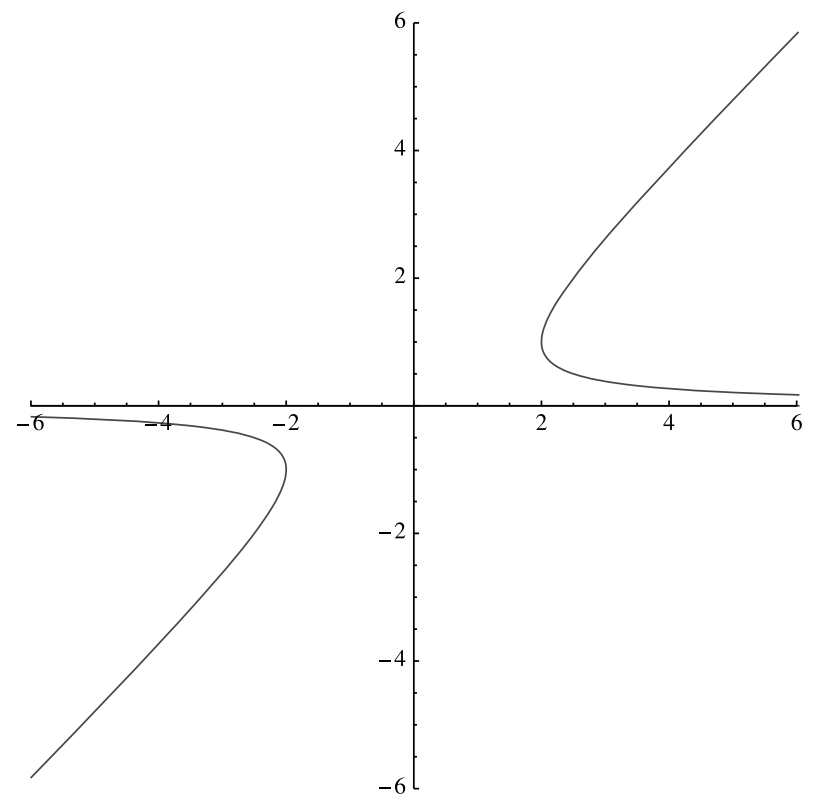

Figure 9: The spectral curve $x=y+\frac{1}{y}$.

(4.2) that

$$
t \rightarrow \infty \quad \Longleftrightarrow \quad \mathrm{e}^{-w}=\frac{t+1}{t-1} \rightarrow 1 \quad \Longleftrightarrow \quad w \rightarrow 0,
$$

and the $w \rightarrow 0$ behavior of the Laplace transform represents the Euclidean volume function, as explained in Section 5. Even though there is a symmetry in the $t$-variables, in terms of $w$, we have

$$
t \rightarrow 0 \quad \Longleftrightarrow \quad \mathrm{e}^{-w}=\frac{t+1}{t-1} \rightarrow-1
$$

and this limit does not correspond to bringing the mesh of the lattice to 0 .

By restricting (7.15) to the top degree terms using

$$
\frac{\left(t^{2}-1\right)^{3}}{t^{2}}=t^{4}-3 t^{2}+3-\frac{1}{t^{2}}
$$

we obtain the recursion for the Euclidean volume (figure 10). 


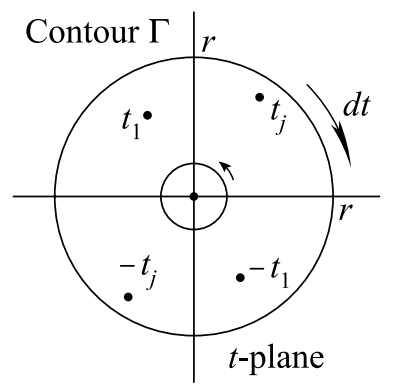

Figure 10: The integration contour $\Gamma$. This contour encloses an annulus bounded by two concentric circles centered at the origin. The outer one has a large radius $r>\max _{j \in N}\left|t_{j}\right|$ and the negative orientation, and the inner one has an infinitesimally small radius with the positive orientation.

Theorem 7.2. Define the Eynard kernel for the Euclidean volume by

$$
K_{\mathrm{E}}\left(t, t_{1}\right)=-\frac{1}{2}\left(\frac{1}{t-t_{1}}+\frac{1}{t+t_{1}}\right) \frac{1}{32} t^{4} \frac{1}{d t} \otimes d t_{1}
$$

on the spectral curve $C_{\mathrm{E}}$ defined by the parametrization

$$
\left\{\begin{array}{l}
x-2=\frac{4}{t^{2}} \\
y-1=\frac{2}{t}
\end{array}\right.
$$

Then the Laplace transformed Euclidean volume function $V_{g, n}^{\mathrm{E}}\left(t_{N}\right)$ satisfies an Eynard-Orantin type recursion

$$
\begin{aligned}
& V_{g, n}^{\mathrm{E}}\left(t_{N}\right) \\
& =-\frac{1}{2 \pi \mathrm{i}} \oint K_{\mathrm{E}}\left(t, t_{1}\right)\left[\sum _ { j = 2 } ^ { n } \left(V_{g, n-1}^{\mathrm{E}}\left(t, t_{N \backslash\{1, j\}}\right) d t \otimes d t_{N \backslash\{1, j\}} \otimes \frac{d s(t) \otimes d t_{j}}{\left(s(t)+t_{j}\right)^{2}}\right.\right. \\
& \left.\quad+V_{g, n-1}^{\mathrm{E}}\left(s(t), t_{N \backslash\{1, j\}}\right) d s(t) \otimes d t_{N \backslash\{1, j\}} \otimes \frac{d t \otimes d t_{j}}{\left(t+t_{j}\right)^{2}}\right) \\
& +V_{g-1, n+1}^{\mathrm{E}}\left(t, s(t), t_{N \backslash\{1\}}\right) d t \otimes d s(t) \otimes d t_{N \backslash\{1\}} \\
& \left.+\sum_{\substack{g_{1}+g_{2}=g \\
I \sqcup J=N \backslash\{1\}}}^{\text {stable }}\left(V_{g_{1},|I|+1}^{\mathrm{E}}\left(t, t_{I}\right) d t \otimes d t_{I}\right) \otimes\left(V_{g_{2},|J|+1}^{\mathrm{E}}\left(s(t), t_{J}\right) d s(t) \otimes d t_{J}\right)\right] .
\end{aligned}
$$

Here the integration contour is a positively oriented circle of large radius. 
The geometry behind the recursion formula (7.22) is the following. The Euclidean volume is obtained by extracting the asymptotic behavior of $\mathcal{L}_{g, n}\left(t_{N}\right)$ as $t \rightarrow \infty$. The parameterization

$$
\left\{\begin{array}{l}
x=2+\frac{4}{t^{2}-1}, \\
y=1+\frac{2}{t-1} .
\end{array}\right.
$$

of the spectral curve (7.11) of figure 9 near $t \sim \infty$ gives a neighborhood of one of the critical points $(x, y)=(2,1)$. Thus we define a new spectral curve $C_{E}$ by the parametrization (7.21), which is simply a parabola $x-2=(y-1)^{2}$. The deck-transformation of the $x$-projection of the parabola $C_{E}$ is still given by $t \mapsto s(t)=-t$. The recipe of (7.9) then gives (7.20), provided that the unstable $(0,2)$ geometry still gives the same kernel

$$
V_{0,2}^{\mathrm{E}}\left(t_{1}, t_{2}\right)=\frac{d t_{1} \otimes d t_{2}}{\left(t_{1}+t_{2}\right)^{2}} .
$$

The continuum limit of (B.7) is

$$
v_{0,2}^{\mathrm{E}}\left(p_{1}, p_{2}\right)=\frac{1}{p_{1}} \delta\left(p_{1}-p_{2}\right) .
$$

We thus calculate

$$
\left(\int_{0}^{\infty} \int_{0}^{\infty} p_{1} p_{2} v_{0,2}^{\mathrm{E}}\left(p_{1}, p_{2}\right) \mathrm{e}^{-\left(p_{1} w_{1}+p_{2} w_{2}\right)} d p_{1} d p_{2}\right) d w_{1} \otimes d w_{2}=\frac{d w_{1} \otimes d w_{2}}{\left(w_{1}+w_{2}\right)^{2}} .
$$

The coordinate change (4.2) near $t \sim \infty$ becomes

$$
\mathrm{e}^{-w}=\frac{t+1}{t-1} \longmapsto 1-w=1+\frac{2}{t},
$$

i.e., $w=-\frac{2}{t}$. Under this change, which is an automorphism of $\mathbb{P}^{1},(7.24)$ remains the same, and we obtain (7.23). The $x$-projection of the spectral curve $C_{E}$ defined by the parametrization (7.21) now has only one ramification point at $t=\infty$. Thus the integration contour $\Gamma$ of (7.15) has changed into a single large circle in (7.22).

The Eynard-Orantin recursion for the symplectic volume is given by the choice of the spectral curve $C_{\mathrm{S}}$ parametrized by

$$
\left\{\begin{array}{l}
x=\frac{1}{t^{2}}, \\
y=\frac{1}{t} .
\end{array}\right.
$$


Since the curve is isomorphic to $\mathbb{C}$, we use the same Cauchy differentiation kernel $W_{02}\left(t_{1}, t_{2}\right)$ of $(7.4)$ in place of $V_{0,2}^{\mathrm{S}}\left(t_{1}, t_{2}\right)$. The Eynard kernel (7.9) for this case is

$$
K\left(t, t_{1}\right)=-\frac{1}{2}\left(-\frac{1}{s(t)+t_{1}}+\frac{1}{t+t_{1}}\right) \frac{t^{4}}{4} \frac{1}{d t} \otimes d t_{1} .
$$

Then the recursion takes exactly the same form of (7.22).

\section{Appendix A. calculation of the Laplace transforms}

In this Appendix we prove the Laplace transform formulas used in the main text. We first derive the topological recursion for

$$
\widehat{L}_{g, n}\left(w_{1}, \ldots, w_{n}\right)=\sum_{\mathbf{p} \in \mathbb{Z}_{\geq 0}^{n}} p_{1} p_{2} \cdots p_{n} N_{g, n}(\mathbf{p}) \mathrm{e}^{-\langle p, w\rangle} .
$$

Since we multiply the number of integral ribbon graphs by $p_{1} \cdots p_{n}$, we can allow all non-negative integers $p_{j}$ in the summation, which makes our calculations simpler.

Proposition A.1. The Laplace transform $\widehat{L}_{g, n}\left(w_{N}\right)$ satisfies the following topological recursion:

$$
\begin{aligned}
\widehat{L}_{g, n}\left(w_{N}\right)= & \sum_{j=2}^{n} \frac{\partial}{\partial w_{j}}\left[\left(\frac{\mathrm{e}^{w_{1}}}{\mathrm{e}^{w_{1}}-\mathrm{e}^{w_{j}}}-\frac{\mathrm{e}^{w_{1}+w_{j}}}{\mathrm{e}^{w_{1}+w_{j}}-1}\right)\right. \\
& \left.\times\left(\frac{\widehat{L}_{g, n-1}\left(w_{N \backslash\{j\}}\right)}{\left(\mathrm{e}^{w_{1}}-\mathrm{e}^{-w_{1}}\right)^{2}}-\frac{\widehat{L}_{g, n-1}\left(w_{N \backslash\{1\}}\right)}{\left(\mathrm{e}^{w_{j}}-\mathrm{e}^{-w_{j}}\right)^{2}}\right)\right] \\
& +\frac{1}{\left(\mathrm{e}^{w_{1}}-\mathrm{e}^{-w_{1}}\right)^{2}}\left[\widehat{L}_{g-1, n+1}\left(w_{1}, w_{1}, w_{N \backslash\{1\}}\right)\right. \\
& \left.+\sum_{\substack{g_{1}+g_{2}=g \\
I \sqcup J=N \backslash\{1\}}}^{\text {stable }} \widehat{L}_{g_{1},|I|+1}\left(w_{1}, w_{I}\right) \widehat{L}_{g_{2},|J|+1}\left(w_{1}, w_{J}\right)\right] .
\end{aligned}
$$

Proof. First we multiply both sides of (3.3) by $p_{2} p_{3} \cdots p_{n}$ and compute its Laplace transform. The left-hand side gives $\widehat{L}_{g, n}\left(w_{N}\right)$. 
The first line of the right-hand side is

$$
\begin{aligned}
& \sum_{j=2}^{n} \sum_{\mathbf{p} \in \mathbb{Z}_{\geq 0}^{n}} \sum_{q=0}^{p_{1}+p_{j}} p_{j} \frac{p_{1}+p_{j}-q}{2}\left[q p_{2} \cdots \widehat{p}_{j} \cdots p_{n} N_{g, n-1}\left(q, p_{N \backslash\{1, j\}}\right)\right] \mathrm{e}^{-\langle p, w\rangle} \\
& =\sum_{j=2}^{n} \sum_{q=0}^{\infty} \sum_{p_{N \backslash\{1, j\}} \in \mathbb{Z}_{\geq 0}^{n-2}}\left[q p_{2} \cdots \widehat{p_{j}} \cdots p_{n} N_{g, n-1}\left(q, p_{N \backslash\{1, j\}}\right)\right] \\
& \quad \times \mathrm{e}^{-\left\langle p_{N \backslash\{1, j\}}, w_{N \backslash\{1, j\}}\right\rangle} \mathrm{e}^{-q w_{1}} \sum_{\ell=0}^{\infty} \ell \mathrm{e}^{-2 \ell w_{1}} \sum_{p_{j}=0}^{q+2 \ell} p_{j} \mathrm{e}^{p_{j}\left(w_{1}-w_{j}\right)},
\end{aligned}
$$

where the symbol ${ }^{\wedge}$ indicates omission of the variable, and we set $p_{1}+$ $p_{j}-q=2 \ell$. Note that $N_{g, n}\left(p_{N}\right)=0$ unless $p_{1}+\cdots+p_{n}$ is even. Therefore, in the Laplace transform we are summing over all $p_{N} \in \mathbb{Z}_{\geq 0}^{n}$ such that $p_{1}+\cdots+p_{n} \equiv 0 \bmod 2$. Since $N_{g, n-1}\left(q, p_{N \backslash\{1, j\}}\right)=0$ unless $q+p_{2}+\cdots+$ $\widehat{p_{j}}+\cdots+p_{n} \equiv 0 \bmod 2$, only those $p_{1}, p_{j}$ and $q$ satisfying $p_{1}+p_{j}-q \equiv 0$ mod 2 contribute in the summation. We now calculate from the last factor (the $p_{j}$-summation)

$$
\begin{aligned}
\sum_{p_{j}=0}^{q+2 \ell} & \mathrm{e}^{-q w_{1}} \ell \mathrm{e}^{-2 \ell w_{1}} p_{j} \mathrm{e}^{p_{j}\left(w_{1}-w_{j}\right)} \\
= & \mathrm{e}^{-q w_{1}} \ell \mathrm{e}^{-2 \ell w_{1}} \frac{\partial}{\partial w_{1}} \frac{\mathrm{e}^{w_{j}}-\mathrm{e}^{w_{j}} \mathrm{e}^{(1+q+2 \ell)\left(w_{1}-w_{j}\right)}}{\mathrm{e}^{w_{j}}-\mathrm{e}^{w_{1}}} \\
= & \frac{\mathrm{e}^{-q w_{1}} \ell \mathrm{e}^{-2 \ell w_{1}}}{\left(\mathrm{e}^{w_{1}}-\mathrm{e}^{w_{j}}\right)^{2}}\left[\mathrm{e}^{w_{1}+w_{j}}+(q+2 \ell) \mathrm{e}^{2 w_{1}} \mathrm{e}^{(q+2 \ell)\left(w_{1}-w_{j}\right)}\right. \\
& \left.-(1+q+2 \ell) \mathrm{e}^{w_{1}+w_{j}} \mathrm{e}^{(q+2 \ell)\left(w_{1}-w_{j}\right)}\right] \\
= & \frac{1}{\left(\mathrm{e}^{w_{1}}-\mathrm{e}^{w_{j}}\right)^{2}}\left[\mathrm{e}^{w_{1}+w_{j}} \mathrm{e}^{-q w_{1}} \ell \mathrm{e}^{-2 \ell w_{1}}+\ell(q+2 \ell) \mathrm{e}^{2 w_{1}} \mathrm{e}^{-q w_{j}} \mathrm{e}^{-2 \ell w_{j}}\right. \\
& \left.-\ell(1+q+2 \ell) \mathrm{e}^{w_{1}+w_{j}} \mathrm{e}^{-q w_{j}} \mathrm{e}^{-2 \ell w_{j}}\right]
\end{aligned}
$$

followed by the $\ell$-summation and then the $q$-summation. We obtain

$$
=\sum_{j=2}^{n}\left[\frac{\mathrm{e}^{w_{1}+w_{j}}}{\left(\mathrm{e}^{w_{1}}-\mathrm{e}^{w_{j}}\right)^{2}}\left(\frac{\widehat{L}_{g, n-1}\left(w_{N \backslash\{j\}}\right)}{\left(\mathrm{e}^{w_{1}}-\mathrm{e}^{-w_{1}}\right)^{2}}-\frac{\widehat{L}_{g, n-1}\left(w_{N \backslash\{1\}}\right)}{\left(\mathrm{e}^{w_{j}}-\mathrm{e}^{-w_{j}}\right)^{2}}\right)\right.
$$




$$
\left.-\frac{\mathrm{e}^{w_{1}}}{\mathrm{e}^{w_{1}}-\mathrm{e}^{w_{j}}} \frac{\partial}{\partial w_{j}} \frac{\widehat{L}_{g, n-1}\left(w_{N \backslash\{1\}}\right)}{\left(\mathrm{e}^{w_{j}}-\mathrm{e}^{-w_{j}}\right)^{2}}\right] .
$$

The second line of (3.3) contributes

$$
\begin{aligned}
& \sum_{j=2}^{n} \sum_{\mathbf{p} \in \mathbb{Z}_{\geq 0}^{n}} H\left(p_{1}-p_{j}\right) \sum_{q=0}^{p_{1}-p_{j}} p_{j} \frac{p_{1}-p_{j}-q}{2} \\
& \times\left[q p_{2} \cdots \widehat{p}_{j} \cdots p_{n} N_{g, n-1}\left(q, p_{N \backslash\{1, j\}}\right)\right] \mathrm{e}^{-\langle p, w\rangle} \\
& =\sum_{j=2}^{n} \sum_{\ell=0}^{\infty} \ell \mathrm{e}^{-2 \ell w_{1}} \sum_{p_{j}=0}^{\infty} p_{j} \mathrm{e}^{-p_{j}\left(w_{1}+w_{j}\right)} \\
& \times \sum_{q=0}^{\infty} \mathrm{e}^{-q w_{1}} \sum_{p_{N \backslash\{1, j\}} \in \mathbb{Z}_{\geq 0}^{n-2}}\left[q p_{2} \cdots \widehat{p_{j}} \cdots p_{n} N_{g, n-1}\left(q, p_{N \backslash\{1, j\}}\right)\right] \\
& \times \mathrm{e}^{-\left\langle p_{N \backslash\{1, j\}}, w_{N \backslash\{1, j\}}\right\rangle} \\
& =\sum_{j=2}^{n} \frac{\mathrm{e}^{w_{1}+w_{j}}}{\left(1-\mathrm{e}^{w_{1}+w_{j}}\right)^{2}} \frac{\widehat{L}_{g, n-1}\left(w_{N \backslash\{j\}}\right)}{\left(\mathrm{e}^{w_{1}}-\mathrm{e}^{-w_{1}}\right)^{2}} .
\end{aligned}
$$

In this calculation we set $p_{1}-p_{j}-q=2 \ell$. Similarly, after putting $p_{j}-p_{1}-$ $q=2 \ell$, the third line of $(3.3)$ yields

$$
\begin{aligned}
- & \sum_{j=2}^{n} \sum_{\mathbf{p} \in \mathbb{Z}_{\geq 0}^{n}} H\left(p_{j}-p_{1}\right) \sum_{q=0}^{p_{j}-p_{1}} p_{j} \frac{p_{j}-p_{1}-q}{2} \\
& \times\left[q p_{2} \cdots \widehat{p_{j}} \cdots p_{n} N_{g, n-1}\left(q, p_{N \backslash\{1, j\}}\right)\right] \mathrm{e}^{-\langle p, w\rangle} \\
= & -\sum_{j=2}^{n} \sum_{q=0}^{\infty} \sum_{\ell=0}^{\infty} \sum_{p_{1}=0}^{\infty}\left(p_{1}+q+2 \ell\right) \ell \mathrm{e}^{-p_{1}\left(w_{1}+w_{j}\right)} \mathrm{e}^{-2 \ell w_{j}} \mathrm{e}^{-q w_{j}} \\
& \times \sum_{p_{N \backslash\{1, j\}} \in \mathbb{Z}_{\geq 0}^{n-2}}\left[q p_{2} \cdots \widehat{p}_{j} \cdots p_{n} N_{g, n-1}\left(q, p_{N \backslash\{1, j\}}\right)\right] \mathrm{e}^{-\left\langle p_{N \backslash\{1, j\}}, w_{N \backslash\{1, j\}}\right\rangle} \\
= & -\sum_{j=2}^{n} \frac{\mathrm{e}^{w_{1}+w_{j}}}{\left(1-\mathrm{e}^{w_{1}+w_{j}}\right)^{2}} \frac{\widehat{L}_{g, n-1}\left(w_{N \backslash\{1\}}\right)}{\left(\mathrm{e}^{w_{j}}-\mathrm{e}^{-w_{j}}\right)^{2}} \\
& +\sum_{j=2}^{n} \frac{\mathrm{e}^{w_{1}}}{\mathrm{e}^{w_{1}}-\mathrm{e}^{-w_{j}}} \frac{\partial}{\partial w_{j}} \frac{\widehat{L}_{g, n-1}\left(w_{N \backslash\{1\}}\right)}{\left(\mathrm{e}^{w_{j}}-\mathrm{e}^{-w_{j}}\right)^{2}} .
\end{aligned}
$$


Summing all contributions, we obtain

$$
\begin{aligned}
\sum_{j=2}^{n}[ & \frac{\mathrm{e}^{w_{1}+w_{j}}}{\left(\mathrm{e}^{w_{1}}-\mathrm{e}^{w_{j}}\right)^{2}}\left(\frac{\widehat{L}_{g, n-1}\left(w_{N \backslash\{j\}}\right)}{\left(\mathrm{e}^{w_{1}}-\mathrm{e}^{-w_{1}}\right)^{2}}-\frac{\widehat{L}_{g, n-1}\left(w_{N \backslash\{1\}}\right)}{\left(\mathrm{e}^{w_{j}}-\mathrm{e}^{-w_{j}}\right)^{2}}\right) \\
& \left.-\frac{\mathrm{e}^{w_{1}}}{\mathrm{e}^{w_{1}}-\mathrm{e}^{w_{j}}} \frac{\partial}{\partial w_{j}} \frac{\widehat{L}_{g, n-1}\left(w_{N \backslash\{1\}}\right)}{\left(\mathrm{e}^{w_{j}}-\mathrm{e}^{-w_{j}}\right)^{2}}\right] \\
& +\sum_{j=2}^{n} \frac{\mathrm{e}^{w_{1}+w_{j}}}{\left(1-\mathrm{e}^{w_{1}+w_{j}}\right)^{2}} \frac{\widehat{L}_{g, n-1}\left(w_{N \backslash\{j\}}\right)}{\left(\mathrm{e}^{w_{1}}-\mathrm{e}^{-w_{1}}\right)^{2}} \\
& -\sum_{j=2}^{n} \frac{\mathrm{e}^{w_{1}+w_{j}}}{\left(1-\mathrm{e}^{w_{1}+w_{j}}\right)^{2}} \frac{\widehat{L}_{g, n-1}\left(w_{N \backslash\{1\}}\right)}{\left(\mathrm{e}^{w_{j}}-\mathrm{e}^{-w_{j}}\right)^{2}} \\
& +\sum_{j=2}^{n} \frac{\mathrm{e}^{w_{1}}}{\mathrm{e}^{w_{1}}-\mathrm{e}^{-w_{j}}} \frac{\partial}{\partial w_{j}} \frac{\widehat{L}_{g, n-1}\left(w_{N \backslash\{1\}}\right)}{\left(\mathrm{e}^{w_{j}}-\mathrm{e}^{-w_{j}}\right)^{2}} \\
= & \sum_{j=2}^{n} \frac{\partial}{\partial w_{j}}\left[\left(\frac{\mathrm{e}^{w_{1}}}{\mathrm{e}^{w_{1}}-\mathrm{e}^{w_{j}}}-\frac{\mathrm{e}^{w_{1}+w_{j}}}{\mathrm{e}^{w_{1}+w_{j}}-1}\right)\right. \\
& \left.\times\left(\frac{\widehat{L}_{g, n-1}\left(w_{N \backslash\{j\}}\right)}{\left(\mathrm{e}^{w_{1}}-\mathrm{e}^{-w_{1}}\right)^{2}}-\frac{\widehat{L}_{g, n-1}\left(w_{N \backslash\{1\}}\right)}{\left(\mathrm{e}^{w_{j}}-\mathrm{e}^{-w_{j}}\right)^{2}}\right)\right] .
\end{aligned}
$$

To compute the Laplace transform of the fourth line of (3.3), we note that

$$
\begin{aligned}
& \frac{1}{2} \sum_{p_{1}=0}^{\infty} \sum_{0 \leq q_{1}+q_{2} \leq p_{1}} q_{1} q_{2}\left(p_{1}-q_{1}-q_{2}\right) \mathrm{e}^{-p_{1} w_{1}} f\left(q_{1}, q_{2}\right) \\
& \quad=\frac{1}{2} \sum_{q_{1}=0}^{\infty} \sum_{q_{2}=0}^{\infty} \sum_{\ell=0}^{\infty} 2 \ell \mathrm{e}^{-2 \ell w_{1}} \mathrm{e}^{-\left(q_{1}+q_{2}\right) w_{1}} q_{1} q_{2} f\left(q_{1}, q_{2}\right) \\
& \quad=\frac{1}{\left(\mathrm{e}^{w_{1}}-\mathrm{e}^{-w_{1}}\right)^{2}} \widehat{f}\left(w_{1}, w_{1}\right),
\end{aligned}
$$

where we set $p_{1}-q_{1}-q_{2}=2 \ell$, and

$$
\widehat{f}\left(w_{1}, w_{2}\right)=\sum_{q_{1}=0}^{\infty} \sum_{q_{2}=0}^{\infty} q_{1} q_{2} f\left(q_{1}, q_{2}\right) \mathrm{e}^{-\left(q_{1} w_{1}+q_{2} w_{2}\right)} .
$$

The reason that $p_{1}-q_{1}-q_{2}$ is even comes from the fact that we are summing over $p_{N} \in \mathbb{Z}_{>0}^{n}$ subject to $p_{1}+\cdots+p_{n} \equiv 0 \bmod 2$, while on the fourth line of (3.3) contributions vanish unless $q_{1}+q_{2}+p_{2}+\cdots+p_{n} \equiv 0 \bmod 2$. 
Therefore we can restrict the summation over those $p_{1}, q_{1}$ and $q_{2}$ subject to $p_{1} \equiv q_{1}+q_{2} \bmod 2$. The calculation of the Laplace transform then becomes straightforward, and the contribution is as in (A.2).

To change from the $w$-coordinates to the $t$-coordinates, we use $(4.2)$ to find

$$
d w_{j}=\frac{2}{t_{j}^{2}-1} d t_{j}, \quad \frac{\partial}{\partial w_{j}}=\frac{t_{j}^{2}-1}{2} \quad \frac{\partial}{\partial t_{j}} .
$$

Each factor changes as follows:

$$
\begin{aligned}
\frac{1}{\left(\mathrm{e}^{w_{j}}-\mathrm{e}^{-w_{j}}\right)^{2}} & =\frac{1}{16} \frac{\left(t_{j}^{2}-1\right)^{2}}{t_{j}^{2}}, \\
\frac{\mathrm{e}^{w_{1}}}{\mathrm{e}^{w_{1}}-\mathrm{e}^{w_{j}}}-\frac{\mathrm{e}^{w_{1}+w_{j}}}{\mathrm{e}^{w_{1}+w_{j}}-1} & =\frac{t_{j}\left(t_{1}^{2}-1\right)}{t_{1}^{2}-t_{j}^{2}}, \\
\widehat{L}_{g, n}\left(w_{1}(t), \ldots, w_{n}(t)\right) & =(-1)^{n} 2^{-n} \mathcal{L}_{g, n}\left(t_{1}, \ldots, t_{n}\right)\left(t_{1}^{2}-1\right) \cdots\left(t_{n}^{2}-1\right) .
\end{aligned}
$$

We can now convert (A.2) to (4.6) by a straightforward calculation.

We now prove Theorem 6.2.

Theorem A.1. The symmetric function $\widehat{V}_{g, n}^{\mathrm{S}}\left(w_{N}\right)$ defined by the Laplace transform

$$
\begin{aligned}
& \widehat{V}_{g, n}^{\mathrm{S}}\left(w_{1}, \ldots, w_{n}\right) d w_{1} \otimes \cdots \otimes d w_{n} \\
& \quad=d_{1} \otimes \cdots \otimes d_{n} \int_{\mathbb{R}_{+}^{n}} v_{g, n}^{\mathrm{S}}(\mathbf{p}) \mathrm{e}^{-\langle w, \mathbf{p}\rangle} d p_{1} \cdots d p_{n}
\end{aligned}
$$

satisfies the topological recursion

$$
\begin{aligned}
\widehat{V}_{g, n}^{\mathrm{S}}\left(w_{N}\right)= & -2 \sum_{j=2}^{\infty} \frac{\partial}{\partial w_{j}}\left[\frac{w_{j}}{w_{1}^{2}-w_{j}^{2}}\left(\frac{\widehat{V}_{g, n-1}^{\mathrm{S}}\left(w_{N \backslash\{j\}}\right)}{w_{1}^{2}}-\frac{\widehat{V}_{g, n-1}^{\mathrm{S}}\left(w_{N \backslash\{1\}}\right)}{w_{j}^{2}}\right)\right] \\
& -\frac{2}{w_{1}^{2}}\left(\widehat{V}_{g-1, n+1}^{\mathrm{S}}\left(w_{1}, w_{1}, w_{N \backslash\{1\}}\right)\right. \\
& \left.+\sum_{\substack{g_{1}+g_{2}=g, I \sqcup J=N \backslash\{1\}}} \widehat{V}_{g_{1},|I|+1}^{\mathrm{S}}\left(w_{1}, w_{I}\right) \widehat{V}_{g_{2},|J|+1}^{\mathrm{S}}\left(w_{1}, w_{J}\right)\right)
\end{aligned}
$$


Proof. Since

$$
\widehat{V}_{g, n}^{\mathrm{S}}\left(w_{N}\right)=(-1)^{n} \int_{\mathbb{R}_{+}^{n}} p_{1} \cdots p_{n} v_{g, n}^{\mathrm{S}}(\mathbf{p}) \mathrm{e}^{-\langle w, \mathbf{p}\rangle} d p_{1} \cdots d p_{n},
$$

we multiply both sides of $(6.6)$ by $(-1)^{n} p_{2} \cdots p_{n}$ and take the Laplace transform. The left-hand side gives $\widehat{V}_{g, n}^{\mathrm{S}}\left(w_{N}\right)$.

For a continuous function $f(q)$, by putting $p_{1}+p_{j}-q=\ell$, we have

$$
\begin{aligned}
\int_{0}^{\infty} & d p_{1} \int_{0}^{\infty} d p_{j} \int_{0}^{p_{1}+p_{j}} d q p_{j} q\left(p_{1}+p_{j}-q\right) f(q) \mathrm{e}^{-\left(p_{1} w_{1}+p_{j} w_{j}\right)} \\
= & \int_{0}^{\infty} d q \int_{0}^{\infty} d \ell \int_{0}^{q+\ell} d p_{j} q \ell f(q) \mathrm{e}^{-q w_{1}} \mathrm{e}^{-\ell w_{1}} p_{j} \mathrm{e}^{p_{j}\left(w_{1}-w_{j}\right)} \\
= & \frac{1}{\left(w_{1}-w_{j}\right)^{2}} \int_{0}^{\infty} d q \int_{0}^{\infty} d \ell q \ell f(q) \\
= & \frac{1}{\left(w_{1}-w_{j}\right)^{2}}\left(\frac{\widehat{f}\left(w_{1}\right)}{w_{1}^{2}}-\frac{\widehat{f}\left(w_{j}\right)}{w_{j}^{2}}\right)-\frac{1}{w_{1}-w_{j}} \frac{\partial}{\partial w_{j}}\left(\frac{\widehat{f}\left(w_{j}\right)}{w_{j}^{2}}\right) \\
= & \frac{\partial}{\partial w_{j}}\left[\frac{1}{w_{1}-w_{j}}\left(\frac{\widehat{f}\left(w_{1}\right)}{w_{1}^{2}}-\frac{\widehat{f}\left(w_{j}\right)}{w_{j}^{2}}\right)\right],
\end{aligned}
$$

where $\widehat{f}(w)=\int_{0}^{\infty} q f(q) \mathrm{e}^{-q w} d q$. By setting $p_{1}-p_{j}-q=\ell$ we calculate

$$
\begin{aligned}
\int_{0}^{\infty} & d p_{1} \int_{0}^{\infty} d p_{j} H\left(p_{1}-p_{j}\right) \int_{0}^{p_{1}-p_{j}} d q p_{j} q\left(p_{1}-p_{j}-q\right) f(q) \mathrm{e}^{-\left(p_{1} w_{1}+p_{j} w_{j}\right)} \\
& =\int_{0}^{\infty} d q \int_{0}^{\infty} d \ell \int_{0}^{\infty} d p_{j} q \ell f(q) \mathrm{e}^{-q w_{1}} \mathrm{e}^{-\ell w_{1}} p_{j} \mathrm{e}^{-p_{j}\left(w_{1}+w_{j}\right)} \\
& =\frac{1}{\left(w_{1}+w_{j}\right)^{2}} \frac{\widehat{f}\left(w_{1}\right)}{w_{1}^{2}}
\end{aligned}
$$

and similarly,

$$
\begin{gathered}
-\int_{0}^{\infty} d p_{1} \int_{0}^{\infty} d p_{j} H\left(p_{j}-p_{1}\right) \int_{0}^{p_{j}-p_{1}} d q p_{j} q\left(p_{j}-p_{1}-q\right) f(q) \mathrm{e}^{-\left(p_{1} w_{1}+p_{j} w_{j}\right)} \\
=-\int_{0}^{\infty} d q \int_{0}^{\infty} d \ell \int_{0}^{\infty} d p_{1} q \ell f(q) \mathrm{e}^{-q w_{j}} \mathrm{e}^{-\ell w_{j}}\left(p_{1}+q+\ell\right) \mathrm{e}^{-p_{1}\left(w_{1}+w_{j}\right)}
\end{gathered}
$$




$$
\begin{aligned}
& =-\int_{0}^{\infty} d q \int_{0}^{\infty} d \ell q \ell f(q) \mathrm{e}^{-q w_{j}} \mathrm{e}^{-\ell w_{j}}\left[\frac{1}{\left(w_{1}+w_{j}\right)^{2}}+\frac{q+\ell}{w_{1}+w_{j}}\right] \\
& =-\frac{1}{\left(w_{1}+w_{j}\right)^{2}} \frac{\widehat{f}\left(w_{j}\right)}{w_{j}^{2}}+\frac{1}{w_{1}+w_{j}} \frac{\partial}{\partial w_{j}}\left(\frac{\widehat{f}\left(w_{j}\right)}{w_{j}^{2}}\right) .
\end{aligned}
$$

Adding (A.5) and (A.6) we obtain

$$
\begin{aligned}
& \int_{0}^{\infty} d p_{1} \int_{0}^{\infty} d p_{j} H\left(p_{1}-p_{j}\right) \int_{0}^{p_{1}-p_{j}} d q p_{j} q\left(p_{1}-p_{j}-q\right) f(q) \mathrm{e}^{-\left(p_{1} w_{1}+p_{j} w_{j}\right)} \\
& -\int_{0}^{\infty} d p_{1} \int_{0}^{\infty} d p_{j} H\left(p_{j}-p_{1}\right) \int_{0}^{p_{j}-p_{1}} d q p_{j} q\left(p_{j}-p_{1}-q\right) f(q) \mathrm{e}^{-\left(p_{1} w_{1}+p_{j} w_{j}\right)} \\
& =\frac{1}{\left(w_{1}+w_{j}\right)^{2}}\left(\frac{\widehat{f}\left(w_{1}\right)}{w_{1}^{2}}-\frac{\widehat{f}\left(w_{j}\right)}{w_{j}^{2}}\right)+\frac{1}{w_{1}+w_{j}} \frac{\partial}{\partial w_{j}}\left(\frac{\widehat{f}\left(w_{j}\right)}{w_{j}^{2}}\right) \\
& =-\frac{\partial}{\partial w_{j}}\left[\frac{1}{w_{1}+w_{j}}\left(\frac{\widehat{f}\left(w_{1}\right)}{w_{1}^{2}}-\frac{\widehat{f}\left(w_{j}\right)}{w_{j}^{2}}\right)\right] .
\end{aligned}
$$

The sum of the right-hand sides of (A.4) to (A.6) thus becomes

$$
\frac{\partial}{\partial w_{j}}\left[\left(\frac{1}{w_{1}-w_{j}}-\frac{1}{w_{1}+w_{j}}\right)\left(\frac{\widehat{f}\left(w_{1}\right)}{w_{1}^{2}}-\frac{\widehat{f}\left(w_{j}\right)}{w_{j}^{2}}\right)\right] .
$$

Therefore, the first three lines of (6.6) yield

$$
-2 \sum_{j=2}^{\infty} \frac{\partial}{\partial w_{j}}\left[\frac{w_{j}}{w_{1}^{2}-w_{j}^{2}}\left(\frac{\widehat{V}_{g, n-1}^{\mathrm{S}}\left(w_{N \backslash\{j\}}\right)}{w_{1}^{2}}-\frac{\widehat{V}_{g, n-1}^{\mathrm{S}}\left(w_{N \backslash\{1\}}\right)}{w_{j}^{2}}\right)\right] .
$$

For a continuous function $f\left(q_{1}, q_{2}\right)$, we have

$$
\begin{aligned}
& \int_{0}^{\infty} d p_{1} \iint_{0 \leq q_{1}+q_{2} \leq p_{1}} q_{1} q_{2}\left(p_{1}-q_{1}-q_{2}\right) f\left(q_{1}, q_{2}\right) \mathrm{e}^{-p_{1} w_{1}} d q_{1} d q_{2} \\
& =\int_{0}^{\infty} d q_{1} \int_{0}^{\infty} d q_{2} \int_{0}^{\infty} d \ell q_{1} q_{2} \ell f\left(q_{1}, q_{2}\right) \mathrm{e}^{-\ell w_{1}} \mathrm{e}^{-\left(q_{1}+q_{2}\right) w_{1}}=\frac{\widehat{f}\left(w_{1}, w_{1}\right)}{w_{1}^{2}}
\end{aligned}
$$

where $\widehat{f}\left(w_{1}, w_{2}\right)=\int_{\mathbb{R}_{+}^{2}} p_{1} p_{2} f\left(p_{1}, p_{2}\right) \mathrm{e}^{-\left(p_{1} w_{1}+p_{2} w_{2}\right)} d p_{1} d p_{2}$. Thus the last two lines of (6.6) give

$$
-\frac{2}{w_{1}^{2}}\left(\widehat{V}_{g-1, n+1}^{\mathrm{S}}\left(w_{1}, w_{1}, w_{N \backslash\{1\}}\right)+\sum_{\substack{g_{1}+g_{2}=g, I \sqcup J=N \backslash\{1\}}} \widehat{V}_{g_{1},|I|+1}^{\mathrm{S}}\left(w_{1}, w_{I}\right) \widehat{V}_{g_{2},|J|+1}^{\mathrm{S}}\left(w_{1}, w_{J}\right)\right) .
$$


This completes the proof of (A.3).

Let us now change the coordinates from $w_{j}$ 's to $t_{j}$ 's that are given by

$$
w_{j}=-\frac{2}{t_{j}}
$$

this time. This change of coordinate gives

$$
d w_{j}=\frac{2}{t_{j}^{2}} d t_{j}, \quad \frac{\partial}{\partial w_{j}}=\frac{t_{j}^{2}}{2} \frac{\partial}{\partial t_{j}} .
$$

Thus the relation in terms of symmetric differential form is

$$
V_{g, n}^{\mathrm{S}}\left(t_{N}\right) d t_{N}=\widehat{V}_{g, n}^{\mathrm{S}}\left(w_{N}\right) d w_{N}
$$

or

$$
V_{g, n}^{\mathrm{S}}\left(t_{N}\right)=2^{n} \frac{\widehat{V}_{g, n}^{\mathrm{S}}\left(w_{N}\right)}{t_{1}^{2} \cdots t_{n}^{2}} .
$$

So we multiply both sides of (A.3) by $\frac{2^{n}}{t_{1}^{2} \cdots t_{n}^{2}}$. From the first term of the first line we obtain

$$
\begin{gathered}
-2 \frac{2^{n}}{t_{1}^{2} \cdots t_{n}^{2}} \sum_{j=2}^{\infty} \frac{\partial}{\partial w_{j}}\left[\frac{w_{j}}{w_{1}^{2}-w_{j}^{2}} \frac{\widehat{V}_{g, n-1}^{\mathrm{S}}\left(w_{N \backslash\{j\}}\right)}{w_{1}^{2}}\right] \\
=-2 \sum_{j=2}^{\infty} \frac{\partial}{\partial t_{j}}\left[\frac{1}{2} \frac{t_{1}^{2} t_{j}}{t_{1}^{2}-t_{j}^{2}} \frac{t_{1}^{2}}{4} V_{g, n-1}^{\mathrm{S}}\left(t_{N \backslash\{j\}}\right)\right] \\
=-\frac{1}{4} \sum_{j=2}^{\infty} \frac{\partial}{\partial t_{j}}\left[\frac{t_{j}}{t_{1}^{2}-t_{j}^{2}} t_{1}^{4} V_{g, n-1}^{\mathrm{S}}\left(t_{N \backslash\{j\}}\right)\right] .
\end{gathered}
$$

Similarly, the second term of the first line becomes

$$
\begin{aligned}
& 2 \frac{2^{n}}{t_{1}^{2} \cdots t_{n}^{2}} \sum_{j=2}^{\infty} \frac{\partial}{\partial w_{j}}\left[\frac{w_{j}}{w_{1}^{2}-w_{j}^{2}} \frac{\widehat{V}_{g, n-1}^{\mathrm{S}}\left(w_{N \backslash\{1\}}\right)}{w_{j}^{2}}\right] \\
& =2 \sum_{j=2}^{\infty} \frac{\partial}{\partial t_{j}}\left[\frac{1}{2} \frac{t_{1}^{2} t_{j}}{t_{1}^{2}-t_{j}^{2}} \frac{t_{j}^{2}}{4} \frac{t_{j}^{2} V_{g, n-1}^{\mathrm{S}}\left(t_{N \backslash\{1\}}\right)}{t_{1}^{2}}\right] \\
& =\frac{1}{4} \sum_{j=2}^{\infty} \frac{\partial}{\partial t_{j}}\left[\frac{t_{j}}{t_{1}^{2}-t_{j}^{2}} t_{j}^{4} V_{g, n-1}^{\mathrm{S}}\left(t_{N \backslash\{1\}}\right)\right] .
\end{aligned}
$$


The second line of (A.3) is easy to convert. This completes the proof of Theorem 6.2.

\section{Appendix B. Examples}

For $(g, n)=(0,3)$, there are three topological shapes of ribbon graphs listed in figure B.1. Cyclic permutations of $\left(p_{1}, p_{2}, p_{3}\right)$ produce different graphs.

Which ribbon graph corresponds to a point $\left(p_{1}, p_{2}, p_{3}\right) \in \mathbb{Z}_{+}^{3}$ depends on which inequality these three numbers satisfy. If $p_{1}>p_{2}+p_{3}$, then the dumbbell shape (figure B.1, bottom left) corresponds to this point. If $p_{1}=$ $p_{2}+p_{3}$, then the shape of $\infty$ (figure B.1, bottom right) corresponds, and if no coordinate is greater than the sum of the other two, then the double circle graph (figure B.1, top) corresponds. These inequalities divide $\mathbb{Z}_{+}^{3}$ into four regions as in figure B.2.
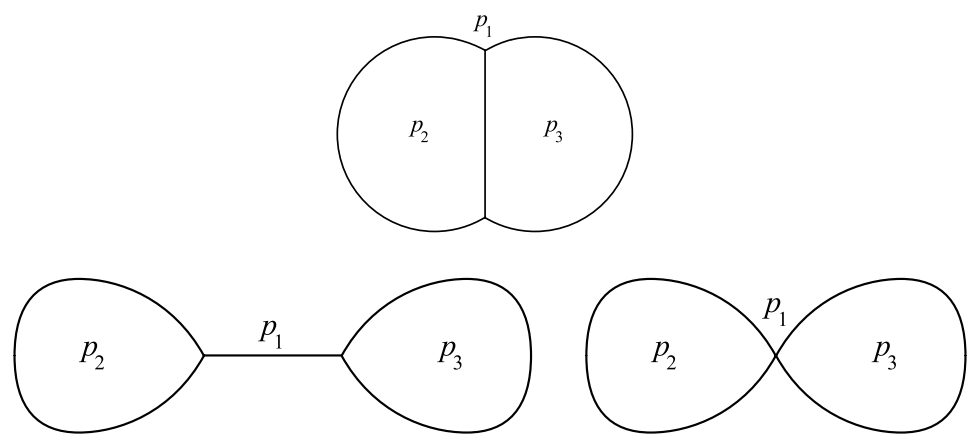

Figure B.1: Three ribbon graphs for $(g, n)=(0,3)$.

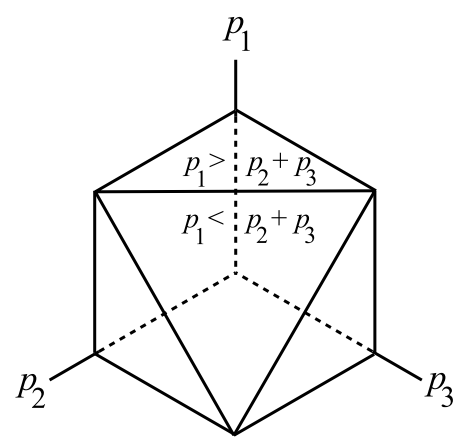

Figure B.2: The partition of $\mathbb{Z}_{+}^{3}$. 
Thus we conclude

$$
N_{0,3}\left(p_{1}, p_{2}, p_{3}\right)= \begin{cases}1 & p_{1}+p_{2}+p_{3} \equiv 0 \quad \bmod 2 \\ 0 & \text { otherwise }\end{cases}
$$

The even parity condition can be met if all three are even or only one of them is even. Let us substitute $p_{j}=2 q_{j}$ when it is even and $p_{j}=2 q_{j}-1$ if it is odd. Thus the Laplace transform can be calculated by

$$
\begin{aligned}
L_{0,3}\left(w_{1}, w_{2}, w_{3}\right)= & \sum_{\left(p_{1}, p_{2}, p_{3}\right) \in \mathbb{Z}_{+}^{3}} N_{0,3}\left(p_{1}, p_{2}, p_{3}\right) \mathrm{e}^{-\left(p_{1} w_{1}+p_{2} w_{2}+p_{3} w_{3}\right)} \\
= & \sum_{\left(q_{1}, q_{2}, q_{3}\right) \in \mathbb{Z}_{+}^{3}}\left(1+\mathrm{e}^{w_{1}+w_{2}}+\mathrm{e}^{w_{2}+w_{3}}+\mathrm{e}^{w_{3}+w_{1}}\right) \\
& \times \mathrm{e}^{-2\left(q_{1} w_{1}+q_{2} w_{2}+q_{3} w_{3}\right)} \\
= & \left(1+\mathrm{e}^{w_{1}+w_{2}}+\mathrm{e}^{w_{2}+w_{3}}+\mathrm{e}^{w_{3}+w_{1}}\right) \\
& \times \frac{\mathrm{e}^{-\left(w_{1}+w_{2}+w_{3}\right)}}{\left(\mathrm{e}^{w_{1}}-\mathrm{e}^{-w_{1}}\right)\left(\mathrm{e}^{w_{2}}-\mathrm{e}^{-w_{2}}\right)\left(\mathrm{e}^{w_{3}}-\mathrm{e}^{-w_{3}}\right)} .
\end{aligned}
$$

Using $\mathrm{e}^{-w_{j}}=\frac{t_{j}+1}{t_{j}-1}$, we obtain

$$
L_{0,3}\left(w\left(t_{1}\right), w\left(t_{2}\right), w\left(t_{3}\right)\right)=-\frac{1}{16}\left(t_{1}+1\right)\left(t_{2}+1\right)\left(t_{3}+1\right)\left(1+\frac{1}{t_{1} t_{2} t_{3}}\right)
$$

and

$$
\mathcal{L}_{0,3}\left(t_{1}, t_{2}, t_{3}\right)=\frac{\partial^{3}}{\partial t_{1} \partial t_{2} \partial t_{3}} L_{0,3}\left(w\left(t_{1}\right), w\left(t_{2}\right), w\left(t_{3}\right)\right)=-\frac{1}{16}\left(1-\frac{1}{t_{1}^{2} t_{2}^{2} t_{3}^{2}}\right) .
$$

For $(g, n)=(1,1)$, there are two ribbon graphs (see figure B.3) corresponding to a hexagonal and a square tiling of the plane. The hexagonal tiling gives a ribbon graph on the left, and the square one on the right is a degeneration obtained by shrinking the horizontal edge to 0 . The automorphism group is $\mathbb{Z} / 6 \mathbb{Z}$ for the degree 3 graph, and $\mathbb{Z} / 4 \mathbb{Z}$ for the degree 4 graph. 

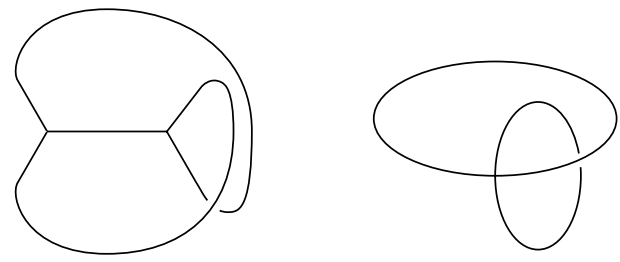

Figure B.3: Two ribbon graphs of type $(1,1)$.

The number of integral ribbon graphs in this case is the number of partitions of the half of the given perimeter length $p=2 q \in 2 \mathbb{Z}_{+}$into two or three positive integers corresponding to edge lengths. Taking the automorphism factors into account, we calculate

$$
N_{1,1}(2 q)=\frac{1}{4}(q-1)+\frac{1}{6} \sum_{r=1}^{q-1}(r-1)=\frac{1}{12}\left(q^{2}-1\right) .
$$

Therefore,

$$
N_{1,1}(p)=\left\{\begin{array}{l}
\frac{1}{48}\left(p^{2}-4\right) \quad p \equiv 0 \quad \bmod 2 \\
0 \quad \text { otherwise }
\end{array}\right.
$$

The Laplace transform can be calculated immediately

$$
L_{1,1}(w)=\sum_{p=2}^{\infty} N_{1,1}(p) \mathrm{e}^{-p w}=\frac{1}{12} \sum_{q=1}^{\infty}\left(q^{2}-1\right) \mathrm{e}^{-2 q w}=\frac{3 \mathrm{e}^{2 w}-1}{12\left(\mathrm{e}^{2 w}-1\right)^{3}}
$$

We thus obtain

$$
L_{1,1}(w(t))=-\frac{1}{384} \frac{(t+1)^{4}}{t^{2}}\left(t-4+\frac{1}{t}\right)
$$

and

$$
\mathcal{L}_{1,1}(t)=-\frac{1}{2^{7}} \frac{\left(t^{2}-1\right)^{3}}{t^{4}}
$$

The values of $(g, n)$ corresponding to genus 0 unstable geometries $(0,1)$, $(0,2)$ play an important role in topological recursion. We have seen this phenomena in Hurwitz theory $[15,38]$. Let us consider the unstable $(0,2)$ case for the integral ribbon graph counting. Although we have restricted our ribbon graphs to have vertices of degree 3 or more, it is indeed more 


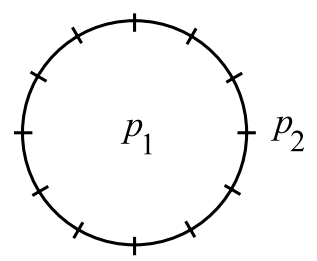

Figure B.4: A ribbon graph of type $(0,2)$.

consistent to allow vertices of degree 2. Actually, a metric ribbon graph with integer edge lengths is a plain ribbon graph whose vertices have degree 2 or more. For such a ribbon graph, we assign length 1 to every edge. We recover a metric ribbon graph with integer edge length by disregarding all vertices of degree 2. This point of view is natural when we assign a Belyi morphism $[1,36,46]$ to a ribbon graph.

Once we allow degree 2 vertices, there is only one kind of ribbon graph of type $(0,2)$, which is a circle (figure B.4). If the graph has $p$ edges, then the automorphism group of this graph is $\mathbb{Z} / p \mathbb{Z}$. Therefore, we have

$$
N_{0,2}\left(p_{1}, p_{2}\right)=\frac{1}{p_{1}} \delta_{p_{1} p_{2}}
$$

and its Laplace transform becomes

$$
L_{0,2}\left(w_{1}, w_{2}\right)=\sum_{p_{1}=1}^{\infty} \sum_{p_{2}=1}^{\infty} N_{0,2}\left(p_{1}, p_{2}\right) \mathrm{e}^{-\left(p_{1} w_{1}+p_{2} w_{2}\right)}=-\log \left(1-\mathrm{e}^{-w_{1}-w_{2}}\right) .
$$

In terms of the $t$-coordinates we have

$$
L_{0,2}\left(w\left(t_{1}\right), w\left(t_{2}\right)\right)=-\log \left(1-\frac{t_{1}+1}{t_{1}-1} \cdot \frac{t_{2}+1}{t_{2}-1}\right)
$$

which gives

$$
\mathcal{L}_{0,2}\left(t_{1}, t_{2}\right)=\frac{1}{\left(t_{1}+t_{2}\right)^{2}}
$$

Note that (B.9) is not a Laurent polynomial and exhibits an exception to the general statement of Theorem 4.1. 
The parametrization of the spectral curve (7.11) defines the $x$-projection map

$$
\pi: \mathbb{P}^{1} \ni t \longmapsto x=\frac{t+1}{t-1}+\frac{t-1}{t+1} \in \mathbb{P}^{1} .
$$

We find that the difference of the Cauchy differentiation kernels of the curve upstairs and downstairs is $\mathcal{L}_{0,2}\left(t_{1}, t_{2}\right)$ :

$$
\mathcal{L}_{0,2}\left(t_{1}, t_{2}\right) d t_{1} \otimes d t_{2}=\frac{d t_{1} \otimes d t_{2}}{\left(t_{1}+t_{2}\right)^{2}}=\frac{d t_{1} \otimes d t_{2}}{\left(t_{1}-t_{2}\right)^{2}}-\pi^{*} \frac{d x_{1} \otimes d x_{2}}{\left(x_{1}-x_{2}\right)^{2}},
$$

where $\pi$ is the $x$-projection map (7.12). We note that this situation is exactly the same as the Hurwitz theory [15, Remark 4.5].

The other genus 0 unstable case $(0,1)$ is important because it identifies the embedding of the spectral curve $(7.11)$ in $\mathbb{C}^{2}$. It is also subtle because we need to allow degree 1 vertices. Since all possible trees can be included if we allow degree 1 vertices, we have to make a choice as to what kind of trees are allowed. For example, we could allow arbitrary trees as in the Hurwitz theory [45]. In the current integral ribbon graph case, we need to make a more restrictive choice. Since this topic has no direct relevance to the main theorems of this paper, we refer to [21] for more details of this matter.

Using the recursion formula (4.6), we can calculate $\mathcal{L}_{g, n}\left(t_{N}\right)$ systematically. A few examples are listed below:

$$
\begin{aligned}
\mathcal{L}_{0,4}\left(t_{1}, t_{2}, t_{3}, t_{4}\right)= & \frac{1}{2^{8}}\left[3 \sum_{j=1}^{4} t_{j}^{2}-9-\sum_{i<j} \frac{1}{t_{i}^{2} t_{j}^{2}}\right. \\
& \left.-\frac{9}{t_{1}^{2} t_{2}^{2} t_{3}^{2} t_{4}^{2}}+\frac{3}{t_{1}^{2} t_{2}^{2} t_{3}^{2} t_{4}^{2}} \sum_{j=1}^{4} \frac{1}{t_{j}^{2}}\right] .
\end{aligned}
$$

$$
\begin{aligned}
& \mathcal{L}_{1,2}\left(t_{1}, t_{2}\right)= \frac{1}{2^{11}}\left[5\left(t_{1}^{4}+t_{2}^{4}\right)+3 t_{1}^{2} t_{2}^{2}-18\left(t_{1}^{2}+t_{2}^{2}\right)+27-4\left(\frac{1}{t_{1}^{2}}+\frac{1}{t_{2}^{2}}\right)\right. \\
&\left.+\frac{27}{t_{1}^{2} t_{2}^{2}}-\frac{18}{t_{1}^{2} t_{2}^{2}}\left(\frac{1}{t_{1}^{2}}+\frac{1}{t_{2}^{2}}\right)+\frac{3}{t_{1}^{4} t_{2}^{4}}+\frac{5}{t_{1}^{2} t_{2}^{2}}\left(\frac{1}{t_{1}^{4}}+\frac{1}{t_{2}^{4}}\right)\right] . \\
& \text { B.13) } \quad \mathcal{L}_{2,1}(t)=-\frac{21}{2^{19}} \frac{\left(t^{2}-1\right)^{7}}{t^{8}}\left(5 t^{2}+6+\frac{5}{t^{2}}\right) .
\end{aligned}
$$


(B.14)

$$
\mathcal{L}_{3,1}(t)=-\frac{11}{2^{30}} \frac{\left(t^{2}-1\right)^{11}}{t^{12}}\left(2275 t^{4}+4004 t^{2}+4722+\frac{4004}{t^{2}}+\frac{2275}{t^{4}}\right) \text {. }
$$

\section{References}

[1] G. V. Belyi, On galois extensions of a maximal cyclotomic fields, Math. U.S.S.R. Izvestija 14 (1980) 247-256.

[2] J. Bennett, D. Cochran, B. Safnuk, and K. Woskoff, Topological recursion for symplectic volumes of moduli spaces of curves, Michigan Math. J., in press.

[3] G. Borot, B. Eynard, M. Mulase and B. Safnuk, Hurwitz numbers, matrix models and topological recursion, arXiv:0906.1206.

[4] V. Bouchard, A. Klemm, M. Mariño and S. Pasquetti, Remodeling the B-model, Commun. Math. Phys. 287 (2008) 117-178.

[5] V. Bouchard and M. Mariño, Hurwitz numbers, matrix models and enumerative geometry, Proc. Symposia Pure Math. 78 (2008) 263-283.

[6] A. Brini, The local Gromov-Witten theory of $\mathbb{C P}^{1}$ and integrable hierarchies, arXiv:1002.0582v1 [math-ph].

[7] L. Chen, Bouchard-Klemm-Marino-Pasquetti Conjecture for $\mathbb{C}^{3}, 2009$, arXiv:0910.3739.

[8] R. Dijkgraaf and C. Vafa, Two dimensional Kodaira-Spencer theory and three dimensional Chern-Simons Gravity, arXiv:0711.1932 [hep-th].

[9] R. Dijkgraaf, E. Verlinde, and H. Verlinde, Loop equations and Virasoro constraints in non-perturbative two-dimensional quantum gravity, Nucl. Phys. B 348 (1991) 435-456.

[10] N. Do and P. Norbury, Counting lattice points in compactified moduli spaces of curves, arXiv:1012.5923 [math.GT].

[11] B. Dubrovin, Geometry of 2D topological field theories, in 'Integrable systems and quantum groups', eds. M. Francaviglia and S. Greco, Montecatini Terme, 1993, 120-348; Lecture Notes in Math. 1620, Springer, Berlin, 1996.

[12] B. Dubrovin and Y. Zhang, Frobenius manifolds and Virasoro constraints, Sel. Math., New Ser. 5, (1999) 423-466. 
[13] B. Eynard, Topological expansion for the 1-hermitian matrix model correlation functions, arXiv:hep-th/0407261.

[14] B. Eynard, Recursion between volumes of moduli spaces, arXiv:0706.4403 [math-ph].

[15] B. Eynard, M. Mulase and B. Safnuk, The Laplace transform of the cut-and-join equation and the Bouchard-Mariño conjecture on Hurwitz numbers, 2009, arXiv:0907.5224 math.AG.

[16] B. Eynard and N. Orantin, Invariants of algebraic curves and topological expansion, Commun. Number Theory Phys. 1, (2007) 347-452.

[17] B. Eynard and N. Orantin, Weil-Petersson volume of moduli spaces, Mirzakhani's recursion and matrix models, arXiv:0705.3600 [math-ph].

[18] H. Fan, T. J. Jarvis, Evan Merrell and Y. Ruan, The Witten equation and its virtual fundamental cycle, 2007, arXiv:0712.4025.

[19] H. Fan, T. J. Jarvis and Y. Ruan, Witten's $D_{4}$ integrable hierarchies conjecture, 2010, arXiv:1008.0927.

[20] H. Fan, T. J. Jarvis and Y. Ruan, The Witten equation and its virtual fundamental cycle, 2007, arXiv:0712.4025.

[21] G. Gliner, M. Mulase and M. Zhou. Catalan numbers and the spectral curve for ribbon graph counting, in preparation.

[22] I.P. Goulden and D.M. Jackson, Transitive factorisations into transpositions and holomorphic mappings on the sphere, Proc. Amer. Math. Soc., 125, (1997) 51-60.

[23] J. L. Harer, The cohomology of the moduli space of curves, in Theory of Moduli, Montecatini Terme, 1985, ed. Edoardo Sernesi, SpringerVerlag, Berlin, 1988, 138-221.

[24] J. L. Harer and D. Zagier, The Euler characteristic of the moduli space of curves, Invent. Math. 85, 457-485 (1986).

[25] Tamás Hausel and Michael Thaddeus, Mirror symmetry, Langlands duality, and the Hitchin system, Invent. Math. 153, (2003) 197-229.

[26] L. Jeffrey and F. Kirwan, Intersection theory on moduli spaces of holomorphic bundles of arbitrary rank on a Riemann surface, Ann. Math. 148, (1998) 109-196. 
[27] L. Jeffrey and J. Weitsman, Toric structures on the moduli space of flat connections on a Riemann surface: volumes and the moment map, Adv. Math. 106, 151-168 (1994).

[28] M. Kazarian, KP hierarchy for Hodge integrals, arXiv:0809.3263.

[29] M. Kazarian, S. Lando, An algebro-geometric proof of Witten's conjecture, J. Amer. Math. Soc. 20, 1079-1089 (2007).

[30] M. Kontsevich, Intersection theory on the moduli space of curves and the matrix Airy function, Commun. Math. Phys. 147, 1-23 (1992).

[31] K. Liu, Heat kernel and moduli space, Math. Res. Lett. 3 (1996) 743-762.

[32] K. Liu and H. Xu, Recursion formulae of higher Weil-Petersson volumes, Int. Math. Res. Not. (5) (2009) 835-859.

[33] M. Mariño, Open string amplitudes and large order behavior in topological string theory, 2006-2008, arXiv:hep-th/0612127.

[34] M. Mirzakhani, Simple geodesics and Weil-Petersson volumes of moduli spaces of bordered Riemann surfaces, Invent. Math. 167 (2007) 179-222.

[35] M. Mirzakhani, Weil-Petersson volumes and intersection theory on the moduli space of curves, J. Amer. Math. Soc. 20 (2007) 1-23.

[36] M. Mulase and M. Penkava, Ribbon graphs, quadratic differentials on Riemann surfaces, and algebraic curves defined over $\overline{\mathbb{Q}}$, Asian J. Math. 2 (4) (1998) 875-920.

[37] M. Mulase and B. Safnuk, Mirzakhani's recursion relations, Virasoro constraints and the KdV hierarchy, Indian J. Math. 50 (2008) $189-228$.

[38] M. Mulase and N. Zhang, Polynomial recursion formula for linear Hodge integrals, Commun. Number Theory Phys. 4 (2010) 267-294.

[39] D. Mumford, Towards an enumerative geometry of the moduli space of curves, Arithmetic and Geometry, Vol. II, 271-328; Progr. Math., 36, Birkhauser Boston, Boston, MA, 1983.

[40] P. Norbury, Counting lattice points in the moduli space of curves, Math. Res. Lett. 17(3), (2010) 467-481.

[41] P. Norbury, String and dilaton equations for counting lattice points in the moduli space of curves, 2009, arXiv:0905.4141. 
[42] P. Norbury, Cell decompositions of moduli space, lattice points and Hurwitz problems, 2010, arXiv:1006.1153.

[43] A. Okounkov, Random matrices and random perputations, Int. Math. Res. Not. 2000 (2000) 1043-1095.

[44] A. Okounkov, Toda equations for Hurwitz numbers, Math. Res. Lett. 7 (2000) 447 [arXiv:math.AG/0004128].

[45] A. Okounkov and R. Pandharipande, Gromov-Witten theory, Hurwitz numbers, and matrix models, I, Proc. Symp. Pure Math. 80 (2009) $325-414$.

[46] L. Schneps and P. Lochak, Geometric Galois actions, London Mathematical Society Lecture Notes Series, 242, 1997.

[47] D. D. Sleator, R. E. Tarjan, and W. P. Thurston, Rotation distance, triangulations, and hyperbolic geometry, J. Amer. Math. Soc. 1 (1988) 647-681.

[48] Kurt Strebel, Quadratic differentials, Springer-Verlag, Berlin, 1984.

[49] A. Strominger, S. T. Yau, and E. Zaslow, Mirror symmetry is T-duality, Nucl. Phys. B 479 (1996) 243-259.

[50] R. Vakil, Harvard Thesis, 1997.

[51] E. Witten, Two dimensional gravity and intersection theory on moduli space, Surv. Differential Geometry 1, 243-310 (1991).

[52] E. Witten, On quantum gauge theories in two dimensions, Commun. Math. Physics 141, 153-209 (1991).

[53] J. Zhou, Local mirror symmetry for one-legged topological vertex, 2009, arXiv:0910.4320.

[54] J. Zhou, Local mirror symmetry for the topological vertex, 2009, arXiv:0911.2343.

Department of Mathematics

University of CALIFORNiA

Los ANGEles, CA 90095, USA

E-mail address: kchapman@math.ucla.edu 
Department of Mathematics

UNIVERSITY OF CALIFORNIA

DAVIS, CA 95616-8633, USA

E-mail address: mulase@math.ucdavis.edu

Department of Mathematics

Central Michigan University

Mount Pleasant, MI 48859, USA

E-mail address: brad.safnuk@cmich.edu

ReCeived February 8, 2011 